homalg

\title{
A META-PACKAGE FOR HOMOLOGICAL ALGEBRA
}

\author{
MOHAMED BARAKAT \& DANIEL ROBERTZ
}

\begin{abstract}
The central notion of this work is that of a functor between categories of finitely presented modules over so-called computable rings, i.e. rings $R$ where one can algorithmically solve inhomogeneous linear equations with coefficients in $R$. The paper describes a way allowing one to realize such functors, e.g. $\operatorname{Hom}_{R}, \otimes_{R}, \operatorname{Ext}_{R}^{i}, \operatorname{Tor}_{i}^{R}$, as a mathematical object in a computer algebra system. Once this is achieved, one can compose and derive functors and even iterate this process without the need of any specific knowledge of these functors. These ideas are realized in the ring independent package homalg. It is designed to extend any computer algebra software implementing the arithmetics of a computable ring $R$, as soon as the latter contains algorithms to solve inhomogeneous linear equations with coefficients in $R$. Beside explaining how this suffices, the paper describes the nature of the extensions provided by homalg.
\end{abstract}

\section{INTRODUCTION}

In the setup of finitely presented module categories, homalg realizes functors as mathematical objects which, up to now, can be composed and derived. To this end it realizes, unlike all present systems, the functors not only by the object part, but automatically also by the morphism part.

homalg is abstractly designed and therefore can be used as an extension of other mathematical software providing the necessary ring arithmetics in any concrete problem.

1.1. homalg as a programming environment for homological algebra. Functors map objects of a source category to objects of a target category and, in a compatible way, morphisms between two objects in the source category to morphisms between their images in the target category. So when one implements a functor one has not only to take care of how it acts on objects, but also of how it acts on morphisms between these objects.

Homological algebraic constructions [CE99, HS97, MR01, Rot79, Wei94] are present in most of the computer algebra systems such as Macaulay 2 [GS], Singular [GPS05]/Plural [LS03], and CoCoA [CoC]. One can often find a procedure, let us call it Hom, to compute $\operatorname{Hom}_{R}(A, B)$ for two finitely presented modules $A$ and $B$ over commutative rings that are implemented in these systems. Now given two further modules $M$ and $N$ and a morphism $M \stackrel{\varphi}{\rightarrow} N$, what about applying $\operatorname{Hom}_{R}(A,-)$ or $\operatorname{Hom}_{R}(-, B)$ to the morphism $\varphi$ ? Mathematically it is clear how $\operatorname{Hom}_{R}(A,-)$ or $\operatorname{Hom}_{R}(-, B)$ induces a morphism $\operatorname{Hom}_{R}(A, M) \stackrel{\operatorname{Hom}_{R}(A, \varphi)}{\longrightarrow} \operatorname{Hom}_{R}(A, N)$ or $\operatorname{Hom}_{R}(N, B) \stackrel{\operatorname{Hom}_{R}(\varphi, B)}{\longrightarrow} \operatorname{Hom}_{R}(M, B)$, but on

Date: 2007. 
the level of a computer implementation the information to compute these induced morphisms is normally not contained in the procedure Hom. So one needs to write or find two completely independent procedures, say HomMap and Hom2Map implementing the computations of the induced morphisms. HomMap is for example essential ${ }^{1}$ if one wants to compute $\operatorname{Ext}_{R}^{i}(A, B):=\mathrm{R}^{i} \operatorname{Hom}_{R}(-, B)(A)$, the $i^{\text {th }}$ right derived functor of $\operatorname{Hom}_{R}(-, B)$ applied to $A$. Again, a procedure, let us call it Ext, implementing for all $i$ the computation of the extension modules $\operatorname{Ext}_{R}^{i}(A, B)$ is normally not of much help when it comes to computing the induced morphisms $\operatorname{Ext}_{R}^{i}(\varphi, B)$ or $\operatorname{Ext}_{R}^{i}(A, \varphi)$. Writing the corresponding procedures, say ExtMap and Ext2Map, requires one to know about the derivation process, and of course to have Hom, HomMap and Hom2Map predefined. So what about composing functors? Consider $\operatorname{Ext}_{R}^{j}\left(\operatorname{Ext}_{R}^{k}(-,-),-\right)$ for example (cf. [Roo62], [Bjö79, p. 58ff]). For the object part one needs to compose the procedure Ext with itself using the first argument. Let us call this compositum ExtExt. The morphism part has now three components. Let us consider $\operatorname{Ext}_{R}^{j}\left(\operatorname{Ext}_{R}^{k}(A,-), B\right)$ for example. Again, a procedure ExtExt is offhand not of much help. For this morphism part one rather needs a predefined Ext2Map to first compute $\psi:=\operatorname{Ext}_{R}^{k}(A, \varphi)$, then a predefined ExtMap to compute $\operatorname{Ext}_{R}^{j}(\psi, B)$. Let us call the resulting procedure ExtExt2Map.

So in order to derive or compose functors, one needs their part on objects but also their part on morphisms. This means, that for each functor one has to implement one procedure for the object part and as many procedures as needed for the morphism part. For more complex functors, constructed out of given ones via iterated compositions and derivations ${ }^{2}$ this quickly becomes unfeasable. So on the level of the computer implementation the following became unavoidable:

- Include the mathematical information of how the bifunctor $\operatorname{Hom}_{R}(-,-)$ acts on morphisms inside the procedure Hom itself. This has to be done in a way, that a general procedure, say FunctorMap, is able to extract this information out of Hom. Further HomMap and Hom2Map should now be defined only using FunctorMap applied to Hom.

- Implement a general right derivation procedure for a contravariant functor (given by both its parts), let us call it RightDerivedCofunctor. Now define Ext only using RightDerivedCofunctor applied to the Hom-functor given by both ${ }^{3}$ its parts [Hom, [HomMap, Hom2Map]]. The procedure Ext, or rather the derivation procedure used to define Ext, should be able to reconstruct the mathematical information of how the bifunctor $\operatorname{Ext}_{R}(-,-)$ acts on morphisms, and this in such a way that the same procedure FunctorMap mentioned above is able to extract this information out of Ext. The derivation procedure should reconstruct this information alone from

\footnotetext{
${ }^{1}$ Maybe not in full generality, since one only needs to apply $\operatorname{Hom}_{R}(-, B)$ to morphisms between free modules $R^{1 \times l_{i}} \stackrel{\varphi}{\rightarrow} R^{1 \times l_{i}^{\prime}}$.

${ }^{2}$ Left derivation for covariant functors and right derivation for contravariant functors (=cofunctors).

${ }^{3}$ Since we right derive $\operatorname{Hom}_{R}(-,-)$ with respect to its first argument, the corresponding morphism procedure HomMap has to be marked in the input of RightDerivedCofunctor. This is a minor technical issue.
} 
its input [Hom, $\overline{\text { HomMap }}$ Hom2Map]]. ExtMap and Ext2Map should now be defined only using FunctorMap applied to Ext.

- Implement a general composition procedure for two functors (given by both their parts), let us call it ComposeFunctors. Then define ExtExt only using ComposeFunctors applied ${ }^{4}$ to [Ext, $\overline{\text { ExtMap }}$ Ext2Map]] and [Ext, [ExtMap, Ext2Map]]. The procedure ExtExt, or rather the composition procedure used to define ExtExt, should be able to reconstruct the mathematical information of how the trifunctor $\operatorname{Ext}_{R}^{j}\left(\operatorname{Ext}_{R}^{k}(-,-),-\right)$ acts on morphisms, in such a way that the same procedure FunctorMap as above is able to extract this information out of ExtExt. The composition procedure should reconstruct this information, alone out of its input, which is here [Ext, [ExtMap, Ext2Map]] and [Ext, [ExtMap, Ext2Map]]. Again, ExtExtMap, ExtExt2Map and ExtExt3Map should now be defined only using FunctorMap applied to ExtExt.

Like FunctorMap, the procedures RightDerivedCofunctor and ComposeFunctors should be implemented in a way that is independent of the functors they are applied to. So, with the same RightDerivedCofunctor one should be able to define $\mathrm{R}^{i} \operatorname{Ext}_{R}^{j}\left(\operatorname{Ext}_{R}^{k}(A,-), B\right)$ and again with the same FunctorMap compute its part on morphisms, etc.

Starting from Section 5 we will try to isolate the mathematical ideas that helped us to realize functors as mathematical objects. That defining more complex functors in both their parts is now an easy, even automatic task is the first defining property of homalg.

1.2. homalg as a meta-package. Not a single algorithm to compute in any sort of rings is implemented in homalg. Rather it is a package built up of homological algebraic definitions and constructions. On the highest levels one finds the construction of connecting homomorphisms and long exact sequences, the processes of composition and derivation of functors and definitions of various specific functors. On lower levels homalg goes all the way down till it reaches two procedures, which are basically the only ones required from any software implementing the ring specific arithmetics of the (not necessarily commutative) ring $R$. In what follows we refer by "ring package" to such software. To describe the two procedures let $K$ be a (left) $R$-submodule of the free module $R^{1 \times n}$ given by finitely many generators. Further let b be an arbitrary element of $R^{1 \times n}$ and $A$ an $R$-submodule of $K$, again given by finitely many generators $\left(A \leq K \leq R^{1 \times n} \ni \mathrm{b}\right)$. Let $\mathrm{M} \in R^{k \times n}$ resp. $\mathrm{N} \in R^{a \times n}$ be the matrix having as rows the generators of $K$ resp. of $A$.

(Z) The procedure DecideZero(b, M):

Effectively decide if $\mathrm{b}$ is an element of $K$ or not. "Effectively" means: In case the element b belongs to $K$, then the procedure DecideZero returns zero and, if asked to, is able to express $\mathrm{b}$ as an $R$-linear combination of the generators of $M$. Otherwise some element $\mathrm{b}^{\prime} \in \mathrm{b}+K$ is returned. This is equivalent to deciding the solvability of the inhomogeneous $R$-linear system $\mathrm{xM}=\mathrm{b}$ and, if asked to, finding a

\footnotetext{
${ }^{4}$ Since we compose $\operatorname{Ext}_{R}(-,-)$ (here with itself) with respect to the first argument, the corresponding morphism ExtMap has to be marked in the first argument of ComposeFunctors.
} 
particular solution $\mathrm{x} \in R^{1 \times k}$, in case one exists. This is simply the straightforward generalization of the ideal membership problem to submodules.

(S) The procedure SyzygiesGenerators (M, N):

Compute a generating set of the $R$-module of solutions of the homogeneous $R$-linear system $\mathrm{xM}=0 \bmod \mathrm{N}$. For $\mathrm{z} \in R^{1 \times n}$ the statement $\mathrm{z}=0 \bmod \mathrm{N}$ means that there exists a $\mathrm{y} \in R^{1 \times a}$, such that $\mathbf{z}=\mathrm{yN}$. One calls every such solution a syzygy among the generators of $K$ modulo $A$.

As the reader may have noticed, it is not required in $(\mathrm{Z})$ that the output $\mathrm{b}^{\prime}$ of DecideZero only depends on $\mathrm{b}+K$, i.e. homalg does not require from DecideZero to provide a normal form modulo $K$ for every element $\mathrm{b} \in R^{1 \times n}$, except for $\mathrm{b} \in K$, where DecideZero must return zero. Deciding if two elements b, $\mathrm{c} \in R^{1 \times n}$ represent the same class modulo $K$, is reduced to checking if $\mathrm{b}-\mathrm{c} \in K$, i.e. if DecideZero $(\mathrm{b}-\mathrm{c}, \mathrm{M})=0$.

In practice however one often solves $(\mathrm{Z})$ by first constructing a different set of generators for $K$ out of the given one, where this new set satisfies certain properties ${ }^{5}$, such that a reduction algorithm with respect to a set of generators having these properties is available. In what follows we call such a set of generators a basis (this should not be confused with a free basis). Normally, such a basis also provides a way to algorithmically solve (S). homalg enables the ring package to specify a procedure called BasisOfModule to compute such a basis. Internally, homalg only uses the procedure DecideZero to perform reductions with respect to such a basis. In case the ring package performs reductions without computing such a basis the procedure BasisOfModule is to be set to the identity procedure. In what follows we will refer to the output $\mathrm{b}^{\prime}$ of DecideZero(b, M) as the reduction of $\mathrm{b}$ modulo $K$ (or modulo M).

Summing up one can say:

(1) homalg is designed to be easily extendable by sparing the user the technical details of homological constructions.

(2) homalg is a meta-package that is designed to easily extend mathematical software implementing ring arithmetics. All what homalg needs from such an implementation are two procedures: One to effectively solve the ideal membership problem (Z) and one to compute a generating set of syzygies, i.e. to solve (S) (cf. [Sch80, CLO92, KR00, BGTV03, DL06]). We will call such rings computable.

We stress the following point: For homalg it is irrelevant how (Z) and (S) are solved. Hence, the irrelevance of explaining, or even mentioning how to solve these two problems for every particular class of rings is a defining property of homalg, namely its second defining property.

In Section 2 we describe the categories homalg is dealing with. In Section 3 we deduce from the two procedures DecideZero and SyzygiesGenerators all the other procedures used in the sequel. After introducing some notation in Section 4 we describe homalg's philosophy of implementing functors in Sections 5 and 6, whereas Section 7 describes how homalg derives functors. Appendix A outlines the various ring packages that have been

\footnotetext{
${ }^{5}$ E.g. a GAUSsian triangular basis in case of fields or an involutive or GRÖBNER basis in case of polynomial rings.
} 
successfully used in connection with homalg. Appendix B includes some comments to the current implementation. Finally, examples are given in Appendix C. All appendices are available at the site of homalg [BR07c].

It goes without saying that we will suppress some technical issues of the package for the sake of mathematical clarity. The best (technical) guide to the package remains its source code, which in nearly all parts can be read as a mathematical text.

\section{Presentations}

Let $R$ be a left noetherian ring with one. Denote by $\mathcal{C}^{\text {mod }}$ the category of finitely generated left $R$-modules, which is a full subcategory of the abelian category of left $R$ modules. And denote by $e^{\text {mat }}$ the category of finite $R$-presentations with objects being finite dimensional matrices over $R$, where one identifies two matrices $\mathrm{M} \in R^{l_{1} \times l_{0}}$ and $\mathrm{M}^{\prime} \in$ $R^{r_{1} \times l_{0}}$ with the same number $l_{0}$ of columns to one object, if $R^{1 \times l_{1}} \mathrm{M}=R^{1 \times r_{1}} \mathrm{M}^{\prime}$, as $R$ submodules of $R^{1 \times l_{0}}$. The set $\operatorname{Mor}_{\mathrm{C}^{\text {mat }}}(\mathrm{M}, \mathrm{L})$ of morphisms between two objects $\mathrm{M} \in R^{l_{1} \times l_{0}}$

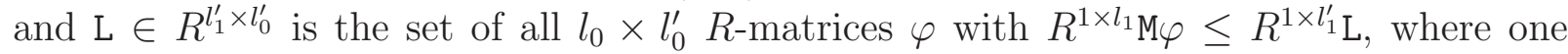
identifies two matrices $\varphi_{1}$ and $\varphi_{2}$ to one morphism, if they induce the same $R$-module homomorphism from $\operatorname{coker}\left(R^{1 \times l_{1}} \stackrel{\mathrm{M}}{\rightarrow} R^{1 \times l_{0}}\right)=R^{1 \times l_{0}} / R^{1 \times l_{1}} \mathrm{M}$ to $\operatorname{coker}\left(R^{1 \times l_{1}^{\prime}} \stackrel{\mathrm{L}}{\rightarrow} R^{1 \times l_{0}^{\prime}}\right)=$ $R^{1 \times l_{0}^{\prime}} / R^{1 \times l_{1}^{\prime} \mathrm{L}}$.

As usual a presentation is given by generators and relations. If one takes the classes of the $l_{0}$ standard basis vectors of $R^{1 \times l_{0}}$ as generators of $M:=\operatorname{coker}\left(R^{1 \times l_{1}} \stackrel{\mathrm{M}}{\rightarrow} R^{1 \times l_{0}}\right)=$ $R^{1 \times l_{0}} / R^{1 \times l_{1}} \mathrm{M}$, then the $l_{1}$ rows of $\mathrm{M}$ are the defining relations between these generators. We thus call $\mathrm{M}$ a presentation matrix or a relation matrix for $M$. Further we call $R^{1 \times l_{1}} \mathrm{M}=\operatorname{im}(\mathrm{M})$ the relation subspace for $M$ generated by (the rows of) $\mathrm{M}$ and denote it by $\langle\mathrm{M}\rangle$.

If $\mathrm{M}$ contains a unit in the $j^{\text {th }}$ column, then the row containing this unit is a relation expressing the $j^{\text {th }}$ generator of $M=\operatorname{coker}(\mathrm{M})$ as an $R$-linear combination in terms of the other generators. Hence one can, using elementary matrix transformations, rewrite the matrix of relations with respect to the remaining generators and obtain a new relation matrix with one less column (and one less row). One iterates this process until the relation matrix is free of units. So, without loss of generality, one can assume that the relation matrix $\mathrm{M}$ is free of units. For deciding invertability of a ring element and computing its inverse homalg uses the procedure Leftinverse described in 3.1.3.

Summing up, the functor coker that maps

$$
R^{l_{1} \times l_{0}} \ni \mathrm{M} \mapsto M:=\operatorname{coker}\left(R^{1 \times l_{1}} \stackrel{\mathrm{M}}{\rightarrow} R^{1 \times l_{0}}\right)
$$

is an equivalence between the categories $\mathcal{C}^{\text {mat }}$ and $\mathrm{C}^{\mathrm{mod}}$. Its inverse functor is to fix a presentation matrix $\mathrm{M} \in \mathrm{Obj}_{\text {emat }}$ for each module $M \in \mathrm{Obj}_{\text {emod }}$ and to use the generators

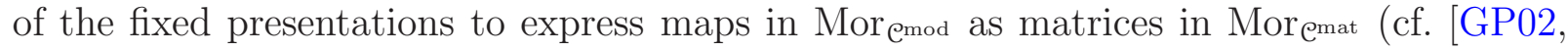
p. 101]).

In what follows we no longer distinguish between the two categories and therefore we simply denote both by $\mathcal{C}$. 


\section{BASIC PROCEDURES}

\subsection{Procedures based on DecideZero.}

3.1.1. RightDivide(B, A, L). This procedure is the ring-theoretic version of finding a particular solution of an inhomogeneous linear system of equations. Hence, it is not astonishing that in a lot of computations it plays the decisive role (cf. 3.1.2-3.1.4).

For three $R$-matrices A, B and L with the same number of columns, one wants to compute an X with

$$
\mathrm{B}=\mathrm{XA} \bmod \mathrm{L},
$$

i.e. an $\mathrm{X}$, such that there exists a $\mathrm{Y}$ with

$$
\mathrm{B}=\mathrm{XA}+\mathrm{YL}=(\mathrm{X}, \mathrm{Y})\left(\begin{array}{l}
\mathrm{A} \\
\mathrm{L}
\end{array}\right) .
$$

After computing (X, Y) one often simply throws away Y. So without loss of generality we forget about $\mathrm{L}$, i.e. consider the system $\mathrm{B}=\mathrm{XA}$. To find $\mathrm{X}$ one computes a basis $\mathrm{G}$ for $\mathrm{A}$ (i.e. a matrix $G$ with rows being a basis for the rows of $A$ ) together with a matrix $C_{A}$, such that $\mathrm{G}=\mathrm{C}_{\mathrm{A}} \mathrm{A}$. Then one reduces $\mathrm{B}$ modulo $\mathrm{G}$, i.e. computes a matrix $\mathrm{N}:=\operatorname{DecideZero}(\mathrm{B}, \mathrm{G})$ (cf. 1.2, (Z)), with rows being those of $\mathrm{B}$ reduced modulo $\mathrm{G}$. We compute $\mathrm{N}$ together with a matrix $C_{B}$, such that $N=B+C_{B} G$. If $N$ is not the zero matrix, then the system is not solvable. Otherwise $\mathrm{X}=-\mathrm{C}_{\mathrm{B}} \mathrm{C}_{\mathrm{A}}$ is a solution. Thinking of $\mathrm{X}$ as " $\mathrm{X}=\mathrm{BA}^{-1}$ " justifies the name of the procedure.

The most prominent application of RightDivide in homalg is the following situation: Given the three modules and the two morphisms

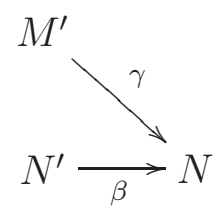

satisfying the so-called image condition $\operatorname{im} \gamma \leq \operatorname{im} \beta$ (as submodules of $N$ ), find a third morphism $\psi: M^{\prime} \rightarrow N^{\prime}$ that makes the triangle commute. The image condition is obviously a necessary condition for such a $\psi$ to exist. There are two instances of special importance for us in which such a $\psi$ always exists:

(1) $M^{\prime}$ is a free module (of finite rank $r$ ), or

(2) $\beta$ is injective.

In the first case let $\left(b_{1}, \ldots, b_{r}\right)$ be a free basis of $M^{\prime}$. Define $b_{i} \psi$ to be any element of the set $\left(b_{i} \gamma\right) \beta^{-1}$, i.e. any element in the preimage of $b_{i} \gamma$ under $\beta$, and since $M^{\prime}$ is free on $\left(b_{1}, \ldots, b_{n}\right)$ this extends by linearity to a morphism $\psi: M^{\prime} \rightarrow N^{\prime}$ satisfying $\psi \beta=\gamma$. In the second case $\beta$ is an isomorphism onto $\operatorname{im} \beta$ and one defines $\psi:=\gamma \beta^{-1}$, where $\gamma$ is viewed as a morphism $M^{\prime} \rightarrow \operatorname{im} \beta$.

Now let $\mathrm{M}^{\prime}, \mathrm{N}^{\prime}$ and $\mathrm{N}$ be relation matrices of $M^{\prime}, N^{\prime}$ and $N$ respectively. Then we are looking for a matrix $\psi$, together with matrices $Y$ and $Z$ such that

(i) $\gamma=\psi \beta+Y \mathrm{~N}$, 
(ii) $\mathrm{M}^{\prime} \psi=Z \mathrm{~N}^{\prime}$.

If one regards $\psi$ merely as a matrix, i.e. not necessarily a morphism $M^{\prime} \rightarrow N^{\prime}$, then condition (i) is nothing else but the above mentioned image condition. Therefore, for the situation described above, such a matrix $\gamma$ always exists. But now condition (ii) states that $\psi$ carries the relations of $M^{\prime}$ to relations of $N^{\prime}$, i.e. it requires $\psi$ to be a morphism from $M^{\prime}$ to $N^{\prime}$. While condition (i) is already in the form needed by RightDivide ${ }^{6}, \psi$ on the left hand side of condition (ii) is multiplied from the right ${ }^{7}$. It now happens that in the two instances (1) and (2) condition (ii) is automatically fulfilled, whenever (i) is. In the first instance one needs to additionally require that $M^{\prime}$ is given on a free basis. Then the matrix of relations $\mathrm{M}^{\prime}$ is the zero matrix (with one row and $r$ columns), so (ii) is trivially satisfied for any $\psi$ by taking $Z$ to be the zero matrix of appropriate dimension. Case (2) is equivalent to saying that the preimage of the subspace $\langle\mathrm{N}\rangle$ of relations for $N$ (generated by $\mathrm{N}$ ) under $\beta$ coincides with the subspace $\left\langle\mathrm{N}^{\prime}\right\rangle$ of relations for $N^{\prime}$ (generated by $\left.\mathrm{N}^{\prime}\right):(\langle\mathrm{N}\rangle) \beta^{-1}=\left\langle\mathrm{N}^{\prime}\right\rangle$. But since $\gamma$ is a morphism $M^{\prime} \rightarrow N$ any relation $m^{\prime} \in\left\langle\mathrm{M}^{\prime}\right\rangle$ of $M$ satisfies $0=m^{\prime} \gamma \stackrel{(\mathrm{i})}{=}\left(m^{\prime} \psi\right) \beta \bmod \langle\mathrm{N}\rangle$. Hence, there exists an $n^{\prime} \in\left\langle\mathrm{N}^{\prime}\right\rangle$, such that $n^{\prime}=m^{\prime} \psi$. So $\psi$ takes relations of $M^{\prime}$ to relations of $N^{\prime}$ and is hence a morphism $M^{\prime} \rightarrow N^{\prime}$.

3.1.2. CompleteImSq. This is the most prominent reincarnation of RightDivide in homalg. We call an incomplete square of the form

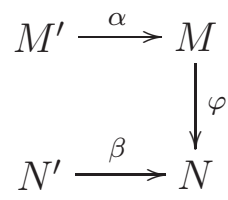

an image square if the image condition $\operatorname{im}(\alpha \varphi) \leq \operatorname{im}(\beta)$ (as submodules of $N$ ) is satisfied. This is precisely the above situation for $\gamma=\alpha \varphi$. In Section 5 CompleteImSq is applied to image squares with injective $\beta$ (case (2)) and in Subsection 7.2 to ones where $M^{\prime}$ is free (case (1)). In these two instances, as shown above, the image square is completable by a morphism $\psi: M^{\prime} \rightarrow N^{\prime}$ which is directly computable using RightDivide.

3.1.3. Leftinverse. The typical applications of Leftinverse correspond to the two situations (1) and (2) described for RightDivide in 3.1.1 (cf. Example C.3):

(1) Either take $M^{\prime}=N$ free (case 3.1.1,(1)), $\gamma=\mathrm{id}$ and $\beta: N^{\prime} \rightarrow N$ surjective. Then the image condition is trivially satisfied. $\psi: M^{\prime}=N \rightarrow N^{\prime}$ is then nothing else but the left inverse or a split of $\beta$.

\footnotetext{
${ }^{6}$ Following the notation used in defining RightDivide we set $\mathrm{A}=\beta, \mathrm{B}=\gamma$ and $\mathrm{L}=\mathrm{N}$.

${ }^{7}$ If the ring $R$ is commutative, one can use the KRONECKER product to construct for any matrix $C$ over $R$ a matrix $\widetilde{C}$ satisfying $\operatorname{row}(C X)=\operatorname{row}(X) \widetilde{C}$ for any composable matrix $X$, where $\operatorname{row}(C)$ is the row vector consisting of the rows of $C$ written behind each other in the obvious order. By this trick one can rewrite the two conditions (i) and (ii) in a single affine condition, where $\operatorname{row}(\psi), \operatorname{row}(Y)$ and $\operatorname{row}(Z)$ are multiplied from the left as required by RightDivide. Since the resulting affine system is, in general, much bigger compared with the initial ones solving it is computationally expensive. Cf. [ZL02].
} 
(2) Or take $M^{\prime}=N, \gamma=\mathrm{id}$ and $\beta: N^{\prime} \rightarrow N$ an isomorphism. Then in particular $\beta$ is injective (case 3.1.1,(2)), and the image condition is trivially satisfied. $\psi: M^{\prime}=$ $N \rightarrow N^{\prime}$ is then nothing else but the (left)inverse of $\beta$.

3.1.4. Preimage. Let $M \stackrel{A}{\rightarrow} L$ be a morphism between the two modules $M$ and $L:=$ coker(L). If the matrix $B$ consists of rows that are in the image of A, i.e. if the image condition is satisfied, then the rows of the matrix $\mathrm{X}$ computed by RightDivide are the preimages of the rows of B under A. In Subsection 7.4 we use the procedure Preimage to construct connecting homomorphisms.

3.2. Procedures additionally based on SyzygiesGenerators. For a matrix $\mathrm{A} \in R^{l_{1} \times l_{0}}$ a matrix of generating syzygies or a syzygy matrix is a matrix $\mathrm{X} \in R^{l_{2} \times l_{1}}$ such that $R^{1 \times l_{2}} \mathrm{X}=$ $\operatorname{ker}\left(R^{1 \times l_{1}} \stackrel{\mathrm{A}}{\rightarrow} R^{1 \times l_{0}}\right)$. A slight generalization of this is when one requires that $R^{1 \times l_{2}} \mathrm{X}=$ $\operatorname{ker}\left(R^{1 \times l_{1}} \stackrel{\mathrm{A}}{\rightarrow} \operatorname{coker}(\mathrm{L})\right)$, where $\mathrm{L} \in R^{a \times l_{0}}$ has the same number $l_{0}$ of columns as A. One then calls $\mathrm{X}$ a syzygy matrix of A modulo $L$, or relative to the module coker $(L)$.

So the rows of the matrix $\mathrm{X}$ of generating syzygies generate the solution space of the homogeneous linear system $\mathrm{xA}=0 \bmod \mathrm{L}$. For $\mathrm{z} \in R^{1 \times l_{0}}$ the statement $\mathrm{z}=0 \bmod \mathrm{L}$ means that there exists a $\mathrm{y} \in R^{1 \times a}$, such that $\mathrm{z}=\mathrm{yL}$.

3.2.1. ResolutionOfModule(M). By iterating the process of taking syzygies one obtains a free resolution of the module $M=\operatorname{coker}(\mathrm{M})$ :

$$
\cdots \stackrel{\varphi_{i+2}}{\longrightarrow} R^{1 \times l_{i+1}} \stackrel{\varphi_{i+1}}{\longrightarrow} R^{1 \times l_{i}} \stackrel{\varphi_{i}}{\longrightarrow} R^{1 \times l_{i-1}} \stackrel{\varphi_{i-1}}{\longrightarrow} \cdots \stackrel{\varphi_{2}}{\longrightarrow} R^{1 \times l_{1}} \stackrel{\varphi_{1}=\mathrm{M}}{\longrightarrow} R^{1 \times l_{0}} \rightarrow M \rightarrow 0
$$

of desired length. What homalg additionally does is the following: As explained in Section 2 we can assume the relation matrix (or the first syzygy matrix) $M=\varphi_{1}$ free of units. Starting from $i=2$, whenever the $i^{\text {th }}$ syzygy matrix $\varphi_{i}$ is computed, homalg uses the units appearing in it to locate the redundant rows of the $(i-1)^{\text {st }}$ syzygy matrix $\varphi_{i-1}$. This means, if $\varphi_{i}$ contains a unit in the $j^{\text {th }}$ column, then the row in $\varphi_{i}$ containing this unit says that the $j^{\text {th }}$ row of $\varphi_{i-1}$ is an $R$-linear combination of the other rows (of $\varphi_{i-1}$ ), so it is redundant for generating the $(i-1)^{\text {st }}$ syzygies and can be omitted from $\varphi_{i-1}$. The $j^{\text {th }}$ row is thus deleted from $\varphi_{i-1}$ and the $i^{\text {th }}$ syzygy matrix $\varphi_{i}$ is recomputed. The rows of the latter are again used to locate further redundant rows of $\varphi_{i-1}$. This process obviously stabilizes. Then one proceeds with the $(i+1)^{\text {st }}$ syzygy matrix $\varphi_{i+1}$, etc. We end up having a free resolution where all the matrices $\varphi_{i}$ are free of units.

In the case of graded modules over a positively graded ring with degree 0 part a field, or modules over local rings, this process indeed yields a minimal free resolution (cf. [Eis95, p. 472] and [Sch03, p. 40]). In both cases eliminating units from the matrices $\varphi_{i}$ of the resolution is equivalent to requiring that all entries belong to the unique maximal graded resp. unique maximal ideal of $R$.

However, and although in general this process does not yield a "minimal" 8 free resolution, it often enough reduces the involved dimensions of the matrices considerably (cf. [KR05]).

\footnotetext{
${ }^{8}$ Such a notion is in general not even well defined, cf. [Eis95, p. 472].
} 
3.2.2. SubfactorModule $\left(\mathrm{M}_{1}, \mathrm{M}_{2}\right)$. Given two matrices $\mathrm{M}_{1}$ and $\mathrm{M}_{2}$ with the same number of columns, computing a presentation matrix for the subfactor module $\left(\left\langle\mathrm{M}_{1}\right\rangle+\left\langle\mathrm{M}_{2}\right\rangle\right) /\left\langle\mathrm{M}_{2}\right\rangle$ (short-hand: $\left.\left\langle\mathrm{M}_{1}\right\rangle /\left\langle\mathrm{M}_{2}\right\rangle\right)$ goes as follows: One computes a basis $\mathrm{B}$ of $\mathrm{M}_{2}$. Then one reduces $\mathrm{M}_{1}$ modulo $\mathrm{B}$ and gets $\mathrm{N}$. The syzygy matrix $\mathrm{S}$ of $\mathrm{M}$ modulo $\mathrm{N}$ is the desired presentation matrix.

\section{Categories of Complexes of given finite Length}

As always, let $\mathcal{C}$ be the module category of Section 2. By $D^{k}(\mathcal{C})$ we denote the category of chain complexes $C: C_{k} \rightarrow C_{k-1} \rightarrow \cdots \rightarrow C_{1}$ of $\mathcal{C}$ of finite length $k-1$ and their chain maps.

Definition 4.1 (The category $D_{i}^{k}(\mathrm{C})$ ). For $i \in\{1, \ldots, k\}$ let $D_{i}^{k}(\mathrm{C})$ be the factor category of $D^{k}(\mathcal{C})$ defined by forgetting in a chain map the morphisms between all but the $i^{\text {th }}$ chain module.

Note that for a morphism in $D_{i}^{k}(\mathcal{C})$

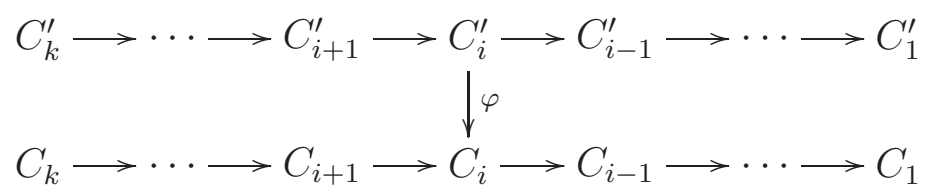

there exists at least one commutative completion

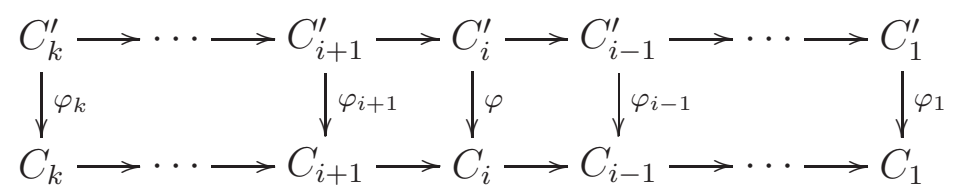

which is a preimage in $D^{k}(\mathcal{C})$.

By definition $D_{1}^{1}(\mathcal{C})$ is simply a different notation for $\mathcal{C}$. From now on all functors we consider take values in the module category $\mathcal{C}$.

Definition 4.2 (The functor $\mathrm{Obj}_{i}^{k}$ ). Denote by $\mathrm{Obj}_{i}^{k}: D_{i}^{k}(\mathrm{C}) \rightarrow \mathcal{C}$ the full functor mapping a complex to its $i^{\text {th }}$ module:

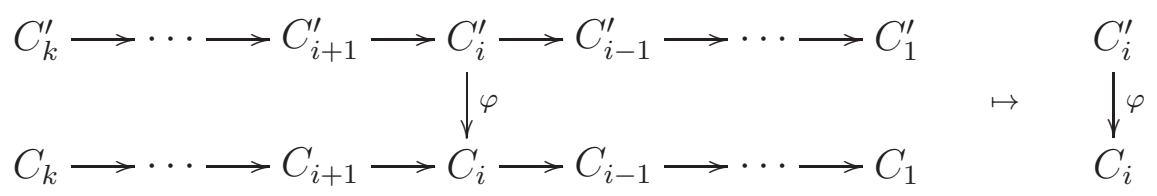

Definition 4.3 (The functor $\operatorname{Mor}_{i}^{k}$ ). Denote by $\operatorname{Mor}_{i}^{k}: D_{i}^{k}(\mathcal{C}) \rightarrow \mathcal{C}$ the full functor mapping a complex to its $(i+1)^{\text {st }}$ morphism:

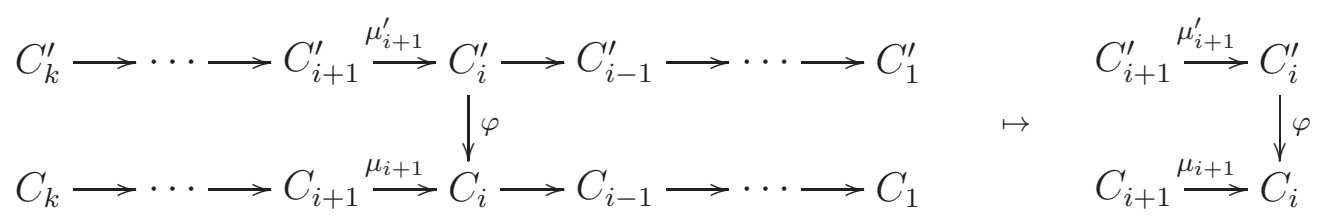




\section{THE MORPHISM PART OF A FUNCTOR}

A functor is by definition a map between two categories that maps objects to objects and, in a compatible way, morphisms to morphisms. To be allowed to speak about functors one needs to be able, not only to define the functor on objects, but also on morphisms between objects.

It might be puzzling for the reader that we start with a section devoted to the morphism part rather than the object part of functors. The reason for that will become clear towards the end of this section.

Here we write everything for covariant functors. Adapting things for contravariant functors is done in the obvious way.

For a morphism $S \stackrel{\varphi}{\rightarrow} T$ and a functor $F$ we want to compute $F(\varphi)$. There are three cases homalg distinguishes:

(Cmp) The functor $F$ is defined in homalg as a composition of two functors: $F=F_{1} \circ F_{2}$.

(Der) The functor $F$ is defined in homalg as the $i^{\text {th }}$ (left) derivation ${ }^{9}$ of another functor: $F=\mathrm{L}_{i} G$.

(Bsc) The functor $F$ is defined in homalg neither by composition nor by derivation. In what follows such functors are called homalg-basic ${ }^{10}$ functors.

Dealing with (Cmp), i.e. with a composed functor is easy: Since $F(\varphi)=F_{1}\left(F_{2}(\varphi)\right)$ one is able to reduce computing $F(\varphi)$ to computing $F_{2}(\varphi)$ and then $F_{1}\left(F_{2}(\varphi)\right.$ ). A bit more involved is the case (Der), i.e. when $F=\mathrm{L}_{i} G$. In Subsection 7.2 it is shown how to reduce the computation of $F(\varphi)$ to essentially computing $G(\varphi)$. For homalg-basic functors the idea is to reduce the computation of $F(\varphi)$ to completing an image square (cf. 3.1.2, case (2)). To this end one embeds $F(S)$ resp. $F(T)$ in a module $H_{u l l}(S)$ resp. $H_{F}(T)$ with an induced morphism $\operatorname{Hull}_{F}(\varphi)$ determined by $\varphi$ :

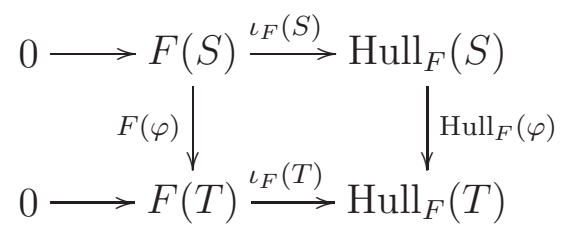

Expressed categorically, $\mathrm{Hull}_{F}$ is a functor, which we call a hull functor of $F$, and $\iota_{F}$ is a natural transformation, which we call the corresponding natural embedding.

For a given functor $F$ the idea is to either define the hull functor Hull $F$ from scratch (e.g. for ${ }^{11}$ Cokernel (in 6.1.1), Hom_R (in 6.2.1), TensorProduct (in 6.2.2) and Hom (in 6.2.3)) or

\footnotetext{
${ }^{9}$ homalg provides procedures to left derive covariant functors and right derive contravariant functors. These are the cases computed via a projective resolution of the module.

${ }^{10}$ The prefix homalg indicates that the notion of "basic functor" is not a mathematical definition. If, for example, the functor $\operatorname{Hom}_{R}\left(\operatorname{Hom}_{R}(-, R), R\right)$ would have been implemented without using homalg's composition procedure ComposeFunctors (see B.4), we then would call it homalg-basic.

${ }^{11}$ Since one can directly provide the action of Cokernel and TensorProduct on morphisms, one takes them as their own hull functors.
} 
to define $\mathrm{Hull}_{F}$ by composition of already defined functors and the forgetful functors $\mathrm{Obj}_{i}^{k}$ (Def. 4.2) and Mor $_{j}^{l}$ (Def. 4.3) (e.g. for Kernel (in 6.1.2) and DefectOfHoms (in 6.1.3)).

The procedure in homalg that precisely accomplishes the above mentioned distinction is called FunctorMap (cf. Appendix B.7).

\section{Some homalg-BASIC FUnCTORS}

Since we need to explain how to construct for each functor its hull functor, we need to first of all mention which specific standard method for computing the object part we use (see for example [GP02]) and then how to construct the hull functor in the specific setup. The hull functor is the missing piece of data that allows one to automatically compute the functor part on morphisms.

All functors we consider are of the form $F: D_{i}^{k}(\mathcal{C}) \rightarrow \mathcal{C}$. We simply distinguish two types of functors, depending on whether $F(C)$ is a subfactor module of $\mathrm{Obj}_{i}^{k}(C)$, for all objects $C \in D_{i}^{k}(\mathcal{C})$, or not. In what follows a functor of the previous kind is called a functor producing subfactor modules.

To avoid confusing the two parts of a functor with source category ${ }^{12} D_{k}^{2}(\mathcal{C})$ (which has as its set of objects also morphisms between modules), we use two different names for the two parts: If the object part of a functor is called $\mathrm{F}$, then its morphism part is called FMap. The name $\mathrm{F}$ is also used to refer to the functor in both its parts.

\subsection{Functors producing subfactor modules.}

6.1.1. The functor Cokernel. On the level of objects the covariant cokernel functor associates to a morphism between two modules its cokernel. Cokernel : $D_{1}^{2}(\mathrm{C}) \rightarrow \mathcal{C}$, i.e.

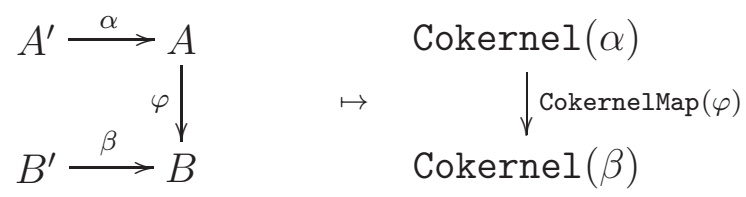

such that

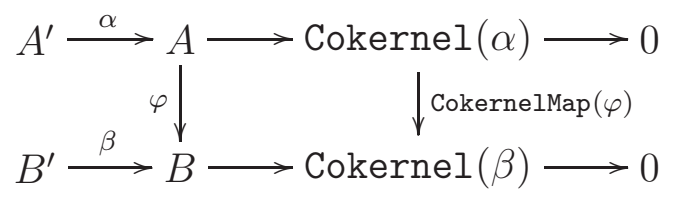

is commutative and exact.

Defining the object part Cokernel $(\alpha)$ is simple: After fixing a presentation matrix A for $A$ and generators for $A^{\prime}$ one can view $\alpha$ as a matrix with the same number of columns as A. Now take the supermatrix $\left(\begin{array}{c}\alpha \\ \mathrm{A}\end{array}\right)$ as a presentation matrix for Cokernel $(\alpha)$.

The cokernel functor is the most basic functor in our setting, in the sense that computing its morphism part CokernelMap $(\varphi)$ is trivial: If we take the residue classes of the generators of $A$ resp. $B$ to be the generators of Cokernel $(\alpha) \operatorname{resp}$. Cokernel $(\beta)$, then

\footnotetext{
${ }^{12}$ This is the case in 6.1.1, 6.1.2.
} 
the matrix representing CokernelMap $(\varphi)$ coincides with that of $\varphi$. Hence one can set the hull functor Hull Cokernel $=$ Cokernel and the natural embedding $\iota_{\text {Cokernel }}$ is the identity transformation $^{13}$.

6.1.2. The functor Kernel. On the level of objects the covariant kernel functor associates to a morphism between two modules its kernel. Kernel : $D_{2}^{2}(\mathrm{C}) \rightarrow \mathrm{C}$, i.e.

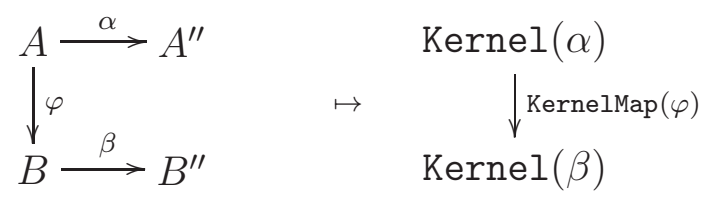

such that

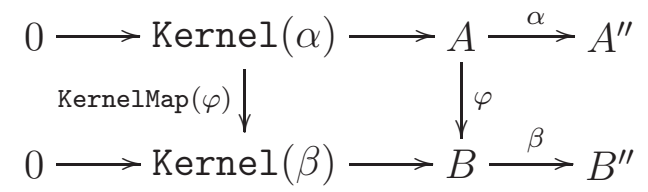

is commutative and exact.

Defining the object part Kernel $(\alpha)$ goes like this: After fixing a presentation matrix A resp. $\mathrm{A}^{\prime \prime}$ for $A$ resp. $A^{\prime \prime}$ one can view $\alpha$ as a matrix with the same number of columns as $A^{\prime \prime}$. Compute the syzygy matrix iota of $\alpha$ modulo $A^{\prime \prime}$ (cf. the beginning of Subsection 3.2). Now use SubfactorModule to compute $\operatorname{Kernel}(\alpha):=\langle$ iota $\rangle /\langle\mathrm{A}\rangle$ (cf. 3.2.2). See also [GP02, p. 97].

In the case of the functor Kernel the hull functor is obviously Hull $\mathrm{Kernel}=\mathrm{Obj}_{2}^{2}$ and the natural embedding $\iota_{\text {Kernel }}$ is the transformation embedding the kernel of a morphism into its source module:

$$
0 \longrightarrow \operatorname{Kernel}(\alpha) \stackrel{\iota_{\text {Kernel }}(\alpha)}{\longrightarrow} A=\mathrm{Obj}_{2}^{2}\left(A \stackrel{\alpha}{\rightarrow} A^{\prime \prime}\right)
$$

The matrix of the natural embedding is simply iota.

6.1.3. The functor DefectOfHoms. On the level of objects the covariant defect functor associates to two composable morphisms $\alpha_{1}$ and $\alpha_{2}$ with $\alpha_{2} \alpha_{1}=0$ their defect of exactness $\operatorname{ker}\left(\alpha_{2}\right) / \operatorname{im}\left(\alpha_{1}\right)$. DefectOfHoms $: D_{2}^{3}(\mathcal{C}) \rightarrow \mathcal{C}$, i.e.
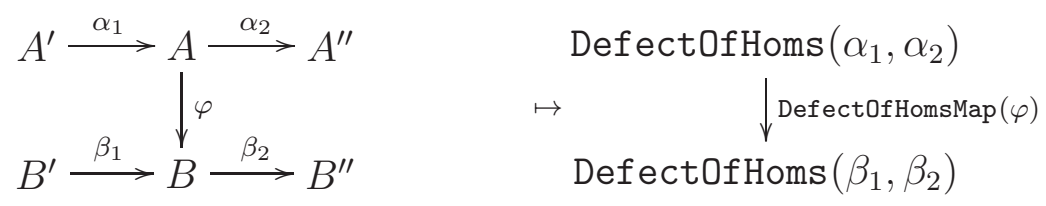

\footnotetext{
${ }^{13}$ This is not the whole truth. The functor Cokernel calls a procedure named Presentation, which, among other things, tries with the help of the procedure BetterGenerators, to reduce the number of generators of the resulting module using either normal form algorithms referred to in the introduction and at the end of Appendix A, in case they are available and applicable, or otherwise, beside the one described in Section 2, several clever heuristics. Taking care of this, is a simple, but technical issue.
} 
such that

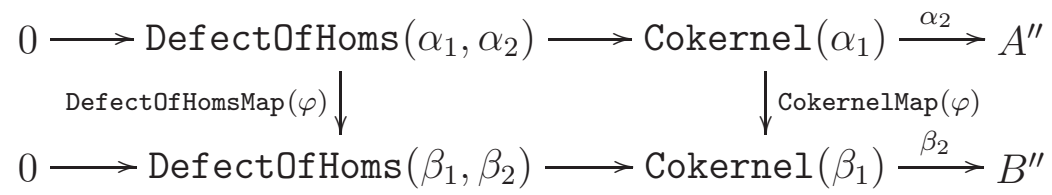

is commutative and exact.

The definition of the object part of Defect0fHoms uses the ideas in 6.1.1 and 6.1.2: Fix a presentation matrix $\mathrm{A}$ for $A$ and $\mathrm{A}^{\prime \prime}$ for $A^{\prime \prime}$. Fix generators for $A^{\prime}$. Then one can view $\alpha_{1}$ resp. $\alpha_{2}$ as a matrix with the same number of columns as A resp. $\mathrm{A}^{\prime \prime}$. Using $\mathrm{A}, \alpha_{2}$ and $\mathrm{A}^{\prime \prime}$ compute the matrix iota of the embedding of Kernel $\left(\alpha_{2}\right)$ in $A$ as in 6.1.2. Then use SubfactorModule to compute the defect DefectOfHoms $\left(\alpha_{1}, \alpha_{2}\right):=\langle\operatorname{iota}\rangle /\left\langle\left(\begin{array}{c}\alpha_{1} \\ \mathrm{~A}\end{array}\right)\right\rangle$.

In the case of the functor DefectOfHoms the hull functor is obviously Hull DefectOfHoms $_{1}=$ Cokernel $\circ \operatorname{Mor}_{2}^{3}$ and the natural embedding $\iota_{\text {DefectofHoms }}$ is the transformation embedding the defect of two composable morphisms into the cokernel of the first morphism:

$$
0 \longrightarrow \text { DefectOfHoms }\left(\alpha_{1}, \alpha_{2}\right) \stackrel{\iota_{\text {DefectofHoms }}\left(\alpha_{1}, \alpha_{2}\right)}{\longrightarrow} \operatorname{Cokernel}\left(\alpha_{1}\right)
$$

where Cokernel $\left(\alpha_{1}\right)=\operatorname{Cokernel}\left(\operatorname{Mor}_{2}^{3}\left(A^{\prime} \stackrel{\alpha_{1}}{\rightarrow} A \stackrel{\alpha_{2}}{\rightarrow} A^{\prime \prime}\right)\right)$. The matrix of the natural embedding is iota.

The functors Cokernel and Kernel are obviously special cases of this functor.

6.2. Other types of homalg-basic functors. Contrary to functors producing subfactor modules, where the morphism part of the hull functor is given by the induced morphism on the factor module, here we need to explicitly mention how the morphism part of the hull functor is defined.

For the rest of the subsection let

$$
M=\operatorname{coker}(\mathrm{M})=R^{1 \times l_{0}} / R^{1 \times l_{1}} \mathrm{M}, \quad L=\operatorname{coker}(\mathrm{L})=R^{1 \times l_{0}^{\prime}} / R^{1 \times l_{1}^{\prime}} \mathrm{L},
$$

be two finitely presented modules:

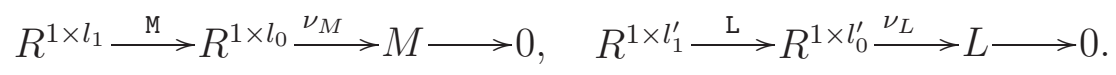

Further let $M \stackrel{\varphi}{\rightarrow} N$ be a morphism, between two finitely presented modules $M$ and

$$
N=\operatorname{coker}(\mathrm{N})=R^{1 \times l_{0}^{\prime \prime}} / R^{1 \times l_{1}^{\prime \prime}} \mathrm{N},
$$

i.e.

$$
\varphi \in R^{l_{0} \times l_{0}^{\prime \prime}}
$$

Note that we write the morphisms on the right, i.e. we use the row convention. 
6.2.1. The functor Hom_R. Let $R$ be a (not necessarily commutative) ring with a fixed involution $^{14} \theta$. For a right $R$-module $H$, define the left $R$-module $H^{\theta}$ by setting $H^{\theta}=H$ as abelian groups and $r \cdot h:=h \theta(r)$ for all $r \in R$ and $h \in H$. So the involution $\theta$ allows one to rewrite any right $R$-module $H$ as a left $R$-module $H^{\theta}$.

Recall that $\operatorname{Hom}_{R}(-, R)$ is a contravariant functor from the category of left $R$-modules to the category of right $R$-modules. We use the involution $\theta$ to transform the resulting right $R$-module into a left $R$-module. We call the resulting functor $\operatorname{Hom}_{R}^{\theta}(-, R)$. The idea is to reduce the computation of the homomorphism module to computing a kernel. Since $\operatorname{Hom}_{R}(-, R)$ is left exact, one obtains the exact sequence of right $R$-modules:

$$
0 \longrightarrow \operatorname{Hom}_{R}(M, R) \stackrel{\nu_{M}^{*}}{\longrightarrow} \operatorname{Hom}_{R}\left(R^{1 \times l_{0}}, R\right) \stackrel{\text { м. }}{\longrightarrow} \operatorname{Hom}_{R}\left(R^{1 \times l_{1}}, R\right) .
$$

We compute $\operatorname{Hom}_{R}(M, R)$ as the kernel of the right most morphism. Following the row convention we identify $\operatorname{Hom}_{R}\left(R^{1 \times j}, R\right)$ with $R^{j \times 1}$, which justifies the notation (M·) for the right most morphism. Applying $\theta$ yields the exact sequence of left $R$-modules:

$$
0 \longrightarrow \operatorname{Hom}_{R}^{\theta}(M, R) \stackrel{\nu_{M}^{*}}{\longrightarrow} R^{1 \times l_{0}} \stackrel{\mathrm{m}^{\theta}}{\longrightarrow} R^{1 \times l_{1}},
$$

where $\left(\mathrm{M}^{\theta}\right)_{a b}=\theta\left(\mathrm{M}_{b a}\right)$ and $\left(R^{l \times 1}\right)^{\theta}=R^{1 \times l}$. Thus set

$$
\operatorname{Hom}_{-} R(M):=\operatorname{Kernel}\left(R^{1 \times l_{0}} \stackrel{\mathrm{M}^{\theta}}{\rightarrow} R^{1 \times l_{1}}\right) .
$$

This definition proposes setting $\operatorname{Hull}_{\text {Hom_R }}(M):=R^{1 \times l_{0}}$ and taking the embedding of the kernel Hom_R(M) in $R^{1 \times l_{0}}$ as the natural embedding (cf. 6.1.2). The morphism part of the hull functor is hence defined by $\operatorname{Hull}_{\text {Hom_R }}(\varphi):=\varphi^{\theta}$ :

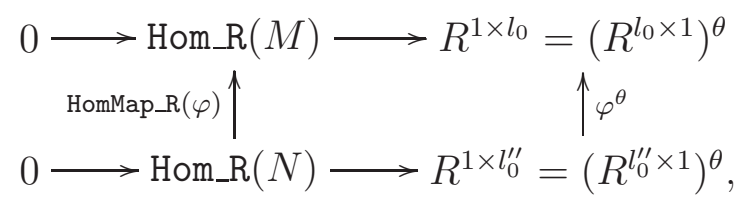

where $\varphi^{\theta}: R^{1 \times l_{0}} \rightarrow R^{1 \times l_{0}^{\prime \prime}}$ is a morphism of free left modules.

Although the definition of $\operatorname{Hull}_{\text {Hom_R }}(M)$ depends on the presentation of $M$, it is nevertheless functorial because of the equivalence of the categories $\mathcal{C}^{\mathrm{mod}}$ and $\mathrm{e}^{\mathrm{mat}}$.

Hom_R is a contravariant functor. The transposition in the definition of $M^{\theta}$ (and $\varphi^{\theta}$ ) is the manifestation of this contravariance.

6.2.2. The functor TensorProduct. Let $R$ be a commutative ${ }^{15}$ ring. Recall that $-\otimes_{R}-$ is a bifunctor, covariant in both arguments. The idea is to reduce the computation of the tensor product module to computing a cokernel. Since $-\otimes_{R}-$ is right exact in both arguments, the tensor product of the two presentations $R^{1 \times l_{1}} \stackrel{\mathrm{M}}{\rightarrow} R^{1 \times l_{0}}$ of $M$ and $R^{1 \times l_{1}^{\prime}} \stackrel{\mathrm{L}}{\rightarrow} R^{1 \times l_{0}^{\prime}}$ of $L$

\footnotetext{
${ }^{14}$ An order 2 anti-automorphism: $\theta^{2}=\operatorname{id}_{R}$ and $\theta(a b)=\theta(b) \theta(a)$ for all $a, b \in R$.

${ }^{15}$ Cf. Subsection 6.2.4.
} 
(each regarded as a two term complex) is a presentation of $M \otimes_{R} L$ (cf. [GP02, Section 2.7]):

$$
\left(R^{1 \times l_{1}} \otimes_{R} R^{1 \times l_{0}^{\prime}}\right) \oplus_{R}\left(R^{1 \times l_{0}} \otimes_{R} R^{1 \times l_{1}^{\prime}}\right) \stackrel{\mathcal{\tau}}{\longrightarrow} R^{1 \times l_{0}} \otimes_{R} R^{1 \times l_{0}^{\prime}} \stackrel{\nu_{M \otimes_{R} L}}{\longrightarrow} M \otimes_{R} L \longrightarrow 0 .
$$

We compute $M \otimes_{R} L$ as the cokernel of $\tau$. After identifying $R^{1 \times j} \otimes_{R} R^{1 \times k}$ with $R^{1 \times j k}$ the morphism $\tau$ is given by the matrix $\mathrm{T}=\left(\begin{array}{c}\mathrm{M} \otimes I_{l_{0}^{\prime}} \\ I_{l_{0}} \otimes \mathrm{L}\end{array}\right)$, where $\otimes$ is the KRONECKER product of matrices and $I_{l_{0}}$ resp. $I_{l_{0}^{\prime}}$ is the identity matrix on $M$ resp. $L$.

Our convention in defining the KRONECKER product of two matrices $A=\left(a_{i j}\right)$ and $B$ is the usual one: $A \otimes B:=\left(a_{i j} B\right)$.

We define

$$
\text { TensorProduct }(M, L):=\operatorname{Cokernel}\left(R^{1 \times\left(l_{1} l_{0}^{\prime}+l_{0} l_{1}^{\prime}\right)} \stackrel{\stackrel{\mathrm{T}}{\rightarrow}}{\rightarrow} R^{1 \times l_{0} l_{0}^{\prime}}\right) .
$$

Define the morphism part of the functor TensorProduct $(-, L)$ to be the KRONECKER product

$$
\text { TensorProduct }(\varphi, L):=\varphi \otimes I_{l_{0}^{\prime}} .
$$

Here the hull functor $\mathrm{Hull}_{\text {TensorProduct }(-, L)}$ coincides with the functor and the natural embedding is the identity transformation.

Analogously for the functor TensorProduct $(L,-)$ define

$$
\text { TensorProduct }(L, \varphi):=I_{l_{0}^{\prime}} \otimes \varphi \text {. }
$$

Again, the hull functor $\mathrm{Hull}_{\text {TensorProduct }(-, L)}$ coincides with the functor and the natural embedding is the identity transformation.

TensorProduct $(-,-)$ is a bifunctor, covariant in each argument.

6.2.3. The functor Hom. Let $R$ be a commutative ${ }^{16}$ ring. Recall that $\operatorname{Hom}_{R}(-,-)$ is a bifunctor, contravariant in its first argument and covariant in the second. The idea is to reduce the computation of the homomorphism module to computing a kernel. Since $\operatorname{Hom}_{R}(-, L)$ is left exact for any module $L$ and $\operatorname{Hom}_{R}(P,-)$ is exact for $P$ projective (or free) one obtains (cf. [GP02, p. 104]):

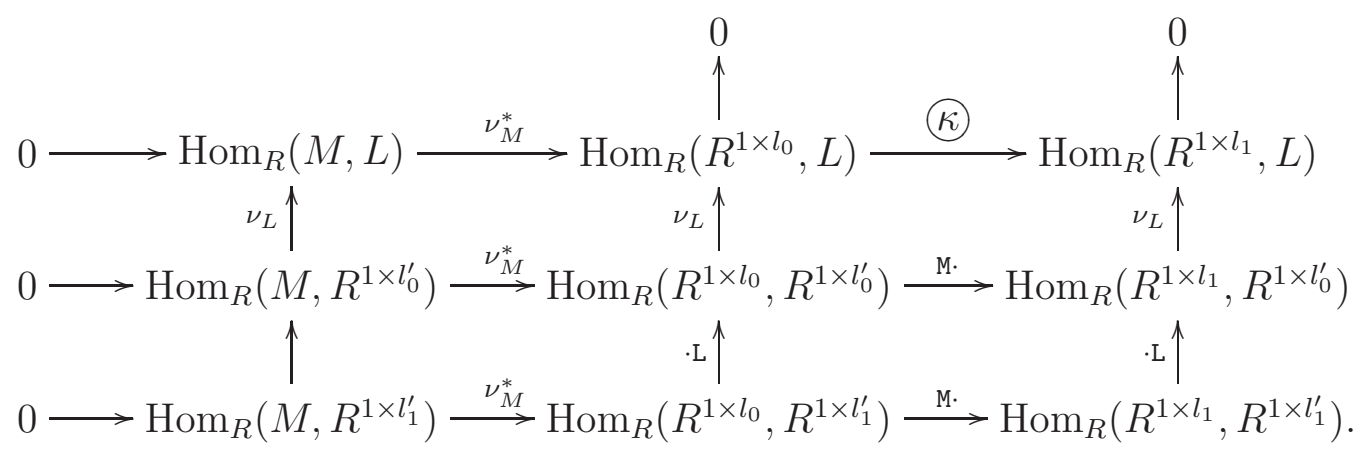

We compute $\operatorname{Hom}_{R}(M, L)$ as the kernel of $\kappa$ in the first row. Identifying $\operatorname{Hom}_{R}\left(R^{1 \times j}, R^{1 \times k}\right)$ with $R^{j \times k}$ justifies the notation used for the morphisms of the lower right square of the

\footnotetext{
${ }^{16}$ Cf. Subsection 6.2.4.
} 
above diagram. Further, identifying $R^{j \times k}$ with $R^{1 \times j k}$ (by writing all the $j$ rows as one long row) gives rise to the identification ${ }^{17}$ of $\operatorname{Hom}_{R}\left(R^{1 \times l_{0}}, L\right)$ with $\operatorname{Cokernel}\left(I_{l_{0}} \otimes \mathrm{L}\right)$ and of $\operatorname{Hom}_{R}\left(R^{1 \times l_{1}}, L\right)$ with Cokernel $\left(I_{l_{1}} \otimes \mathrm{L}\right)$. The induced morphism

$$
\kappa: \operatorname{Hom}_{R}\left(R^{1 \times l_{0}}, L\right) \rightarrow \operatorname{Hom}_{R}\left(R^{1 \times l_{1}}, L\right)
$$

is then given by the matrix $\left(\mathrm{M} \otimes I_{l_{0}^{\prime}}\right)^{\mathrm{tr}}$. Thus define

$$
\operatorname{Hom}(M, L):=\operatorname{Kernel}\left(\operatorname{Cokernel}\left(I_{l_{0}} \otimes \mathrm{L}\right) \stackrel{\left(\mathrm{M} \otimes I_{l_{0}^{\prime}}\right)^{\mathrm{tr}}}{\longrightarrow} \operatorname{Cokernel}\left(I_{l_{1}} \otimes \mathrm{L}\right)\right) .
$$

This definition proposes setting $\operatorname{Hull}_{\mathrm{Hom}(-, L)}(M):=\operatorname{Cokernel}\left(I_{l_{0}} \otimes \mathrm{L}\right)$ and taking the embedding of the kernel $\operatorname{Hom}(M, L)$ in Cokernel $\left(I_{l_{0}} \otimes \mathrm{L}\right)$ as the natural embedding (cf. 6.1.2). The morphism part of the hull functor is hence defined by $\operatorname{Hull}_{\mathrm{Hom}(-, L)}(\varphi):=\left(\varphi \otimes I_{l_{0}^{\prime}}\right)^{\operatorname{tr}}$ :

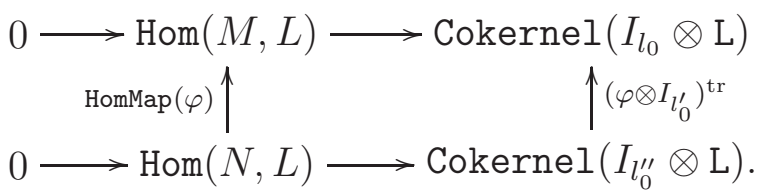

To address the functoriality of $\operatorname{Hom}(-,-)$ in the second argument, interchange the role of $M$ and $L$, and set $\operatorname{Hull}_{\text {Hom }(L,-)}(M):=\operatorname{Cokernel}\left(I_{l_{0}^{\prime}} \otimes \mathrm{M}\right)$ and $\operatorname{Hull}_{\mathrm{Hom}(L,-)}(\varphi):=I_{l_{0}^{\prime}} \otimes \varphi$ :

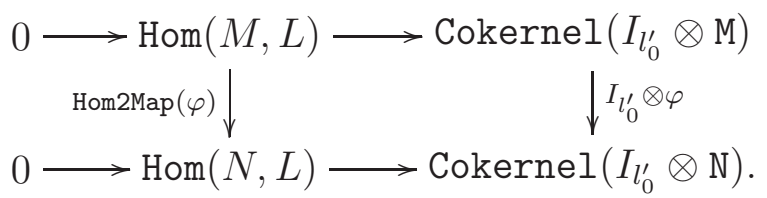

$\operatorname{Hom}(-,-)$ is a bifunctor, contravariant in its first and covariant in its second argument. Transposing the matrix $\mathrm{M} \otimes I_{l_{0}^{\prime}}\left(\right.$ and $\left.\varphi \otimes I_{l_{0}^{\prime}}\right)$ is the manifestation of the contravariance in the first argument.

6.2.4. One last word on the commutativity of the ring $R$. For the most general definition of tensor product of modules one starts with a not necessarily commutative ring $R$, a right $R$-module $M_{R}$, and a left $R$-module ${ }_{R} N$. If the $R$-module structure of $M$ resp. $N$ comes from a $(Q, R)$-bimodule resp. an $(R, S)$-bimodule structure, then their tensor product over $R$ is in a natural way a $(Q, S)$-bimodule ${ }_{Q} M_{R} \otimes_{R} R_{R} N_{S}$ Q and $S$, again, are not necessarily commutative rings. Analogously, let $M$ and $N$ be two left modules over a not necessarily commutative ring $R$ and denote by $\operatorname{Hom}_{R}\left({ }_{R} M,{ }_{R} N\right)$ the abelian group of $R$-homomorphisms. If the $R$-module structure of $M$ resp. $N$ comes from an $(R, Q)$ bimodule resp. an $(R, S)$-bimodule structure, then $\operatorname{Hom}_{R}\left({ }_{R} M,{ }_{R} N\right)$ is again in a natural way a $(Q, S)$-bimodule.

Note that in both cases the resulting module might not be finitely generated as a $(Q, S)$ bimodule, even if $M$ and $N$ are finitely generated as $R$-modules.

${ }^{17} R^{j \times l_{1}^{\prime} \stackrel{\cdot \mathrm{L}}{\longrightarrow}} R^{j \times l_{0}^{\prime}}$ becomes $R^{1 \times j l_{1}^{\prime}} \stackrel{I_{j} \otimes \mathrm{L}}{\longrightarrow} R^{1 \times j l_{0}^{\prime}}$, and $R^{l_{0} \times k} \stackrel{\mathrm{M} \cdot}{\longrightarrow} R^{l_{1} \times k}$ becomes $R^{1 \times l_{0} k} \stackrel{\left(\mathrm{M} \otimes I_{k}\right)^{\mathrm{tr}}}{\longrightarrow} R^{1 \times l_{1} k}$. 
In the special case where either $M$ or $N$ is an $(R, R)$-bimodule, then $M \otimes_{R} N$ resp. $\operatorname{Hom}_{R}(M, N)$ is again an $R$-module ${ }^{18}$. This is always the case when the ring is commutative, since then every $R$-module is an $(R, R)$-bimodule in the obvious way.

The Maple implementation of homalg does neither support changing the ring, nor bimodule structures. These are issues we want to address in future implementations. But even though, the above mentioned problem of non-finite generation will remain the major obstacle.

\section{Derived FunCtors}

The philosophy of derived categories is, roughly speaking, to replace a module by one of its resolutions, and then to look at the resolution as a very special type of complexes, with homology concentrated at degree 0. After inverting quasi-isomorphisms, one obtains the derived category, where the objects are quasi-isomorphism types of complexes. Especially, all resolutions of a module become isomorphic objects in the derived category.

Using the cylinder-cone-translation construction [GM03, III.3] one constructs out of every short exact sequence of complexes, a so called distinguished triangle. By passing to homology we again obtain distinguished triangles in the category of graded objects (which one can view as cyclic complexes, i.e. complexes with zero boundary maps [GM03, III.2.3]). A popular way to start, is to take a distinguished triangle coming from a short exact sequence of complexes that are simultaneously resolving a short exact sequence of modules. Then one applies a functor, that turns such distinguished triangles again into distinguished triangles, and at last one takes the homology. The classical way of writing such a distinguished triangle of homologies is as a long exact homology sequence.

The reason for recalling the standard definitions in the following subsections is not only to indicate how they are computed using homalg, but to finish the discussion of Section 5 . This is done in Subsection 7.2.

7.1. The procedure ResolveModule: Resolve a module. By resolving a module $M$, which we view as a complex concentrated in degree 0, we obtain a complex of free (resp. projective) modules and a quasi-isomorphism

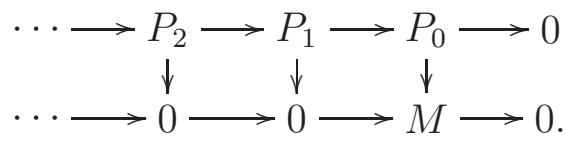

After inverting quasi-isomorphisms all resolutions become isomorphic.

For an additive functor $F$ and a module $M$ with a resolution

$$
P: P_{q+1} \longrightarrow P_{q} \longrightarrow P_{q-1} \longrightarrow \cdots \longrightarrow P_{1} \longrightarrow P_{0} \longrightarrow 0
$$

define the $q^{\text {th }}$ derived functor $\mathrm{L}_{q} F$ applied to $M$ by setting $\mathrm{L}_{q} F(M):=H_{q}(F(P))$, the defect of the two consecutive morphisms $F\left(P_{q+1}\right) \rightarrow F\left(P_{q}\right)$ and $F\left(P_{q}\right) \rightarrow F\left(P_{q-1}\right)$ (cf. 6.1.3 DefectOfHoms).

\footnotetext{
${ }^{18}$ In theory, this special case could work in homalg, and indeed it does for very special cases, of course, beside the trivial case when $M=R$ or $N=R$.
} 
There is a cheaper method to compute the left derivation of a right exact covariant functor, which is based on [HS97, the definition of $\widetilde{\mathrm{L}}_{q}$ in IV.(10.1), p. 156 and Prop. IV.5.5, p. 133] and uses Kernel instead of Defect0fHoms. By duality, there is a cheaper method to compute the right derivation of a left exact contravariant functor, which is based on [HS97, Prop. IV.5.8] and uses Cokernel instead of DefectOfHoms. Both methods are implemented in homalg.

7.2. The procedure ResolutionOfSeq: Resolve a morphism. Given a morphism $M \stackrel{\varphi}{\rightarrow} N$ one resolves $M$ and $N$ freely

$$
\begin{aligned}
& P_{q+1} \longrightarrow P_{q} \longrightarrow P_{q-1} \longrightarrow \cdots \longrightarrow P_{1} \longrightarrow P_{0} \longrightarrow M \longrightarrow 0 \\
& \downarrow \varphi_{q} \quad \downarrow \varphi_{q-1} \quad \downarrow \varphi_{1} \quad \downarrow \varphi_{0} \quad \downarrow \varphi \\
& P_{q+1}^{\prime} \longrightarrow P_{q}^{\prime} \longrightarrow P_{q-1}^{\prime} \longrightarrow \cdots \longrightarrow P_{1}^{\prime} \longrightarrow P_{0}^{\prime} \longrightarrow N \longrightarrow 0 \text {, }
\end{aligned}
$$

and computes the $\varphi_{q}$ 's by iteratively completing image squares. Here one needs a free resolution of $M$ to be in case (2) of CompleteImSq, 3.1.2. Applying $F$ one gets $F\left(P_{q}\right) \stackrel{F\left(\varphi_{q}\right)}{\longrightarrow}$ $F\left(P_{q}^{\prime}\right)$. But now the object part of the functor $\mathrm{L}_{q} F$ applied to $M$ (resp. $N$ ) is a subfactor module of $F\left(P_{q}\right)$ (resp. $F\left(P_{q}^{\prime}\right)$ ) and one is in the situation of DefectOfHoms, 6.1.3. This is all what FunctorMap needs to compute the morphism part of a derived functor. This finishes the discussion of Section 5 .

7.3. The procedure ResolveShortExactSeq: Resolving a short exact sequence of modules. To resolve a short exact sequence of modules $0 \rightarrow M^{\prime} \rightarrow M \rightarrow M^{\prime \prime} \rightarrow 0$, one starts with a resolution of $M^{\prime \prime}$ :

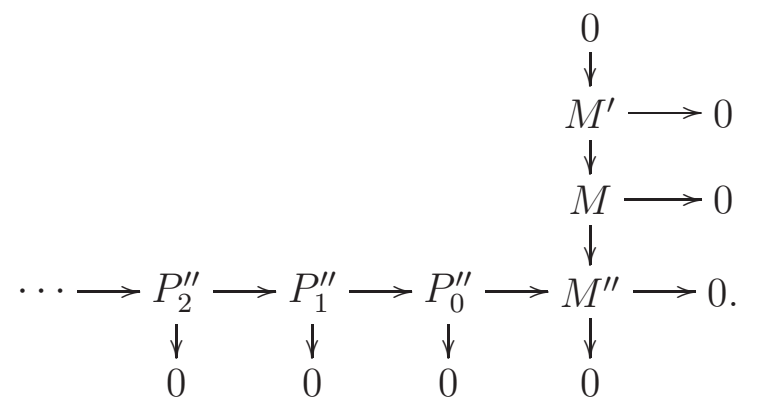

Then one completes the middle line by taking free hulls of iterated pullbacks. Finally one fills the upper line by taking kernels to obtain:

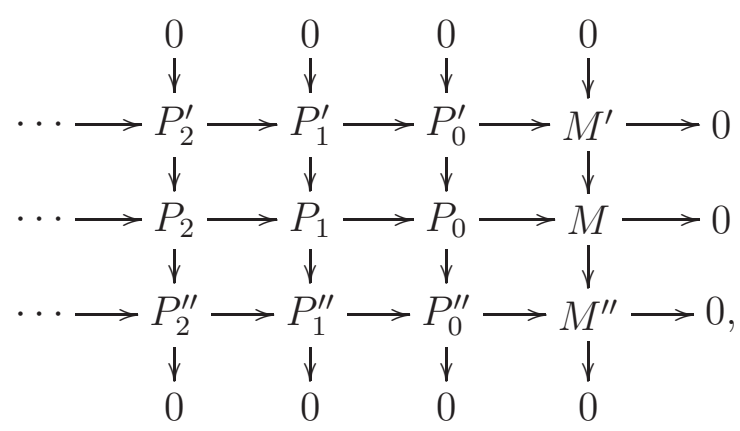


with exact columns and rows. This method is implemented in the procedure ResolveShortExactSeq. There are several other methods to resolve a short exact sequence simultaneously, cf. [HS97, Proof of Theorem IV.6.1].

Computing the pullback $A^{\prime}$ of $B^{\prime} \stackrel{\beta}{\rightarrow} B \stackrel{\varphi}{\leftarrow} A$ is reduced to computing a kernel, namely that of $A \oplus B^{\prime} \stackrel{\left(\begin{array}{c}\varphi \\ -\beta\end{array}\right)}{\longrightarrow} B$. The two maps $B^{\prime} \stackrel{\psi}{\leftarrow} A^{\prime} \stackrel{\alpha}{\rightarrow} A$ of the pullback are the two parts of the kernel embedding $0 \rightarrow A^{\prime} \stackrel{\left(\begin{array}{ll}\alpha & \psi\end{array}\right)}{\longrightarrow} A \oplus B^{\prime}$ (cf. 6.1.2).

7.4. The procedure LongExactHomologySeq: Connecting homomorphism and long exact sequences. Applying an additive covariant functor $F$ to the truncation of the diagram $(M)$

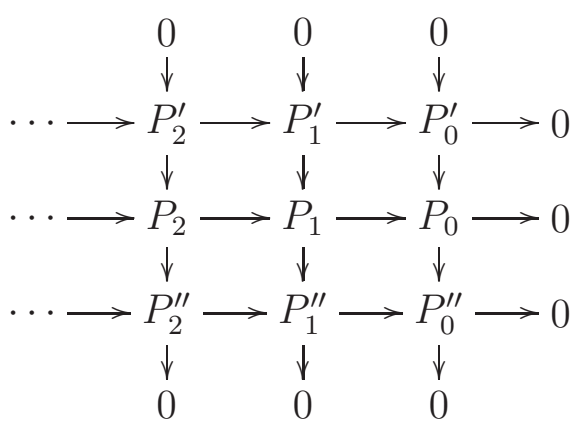

results in a diagram $\bar{P}=F(P)$ where the columns are still exact, but where the rows are now in positive degrees, in general, no longer exact. Connecting the homologies of the rows one obtains the long exact homology sequence

$$
\cdots \stackrel{\partial_{q+1}}{\rightarrow} \mathrm{L}_{q} F\left(M^{\prime}\right) \rightarrow \mathrm{L}_{q} F(M) \rightarrow \mathrm{L}_{q} F\left(M^{\prime \prime}\right) \stackrel{\partial_{q}}{\rightarrow} \mathrm{L}_{q-1} F\left(M^{\prime}\right) \rightarrow \cdots,
$$

where the connecting homomorphisms are computed via the snake lemma applied to the diagrams

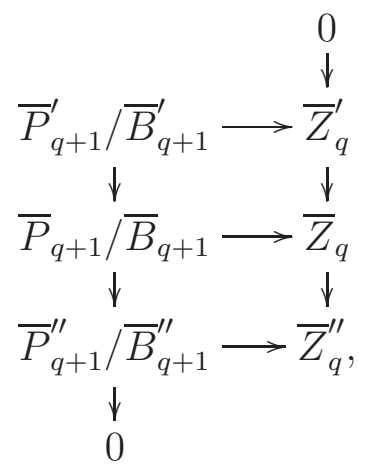

by taking kernels and cokernels, where $\bar{B}_{i}:=\operatorname{im}\left(\bar{P}_{i+1} \rightarrow \bar{P}_{i}\right)$ and $\bar{Z}_{i}:=\operatorname{ker}\left(\bar{P}_{i} \rightarrow\right.$ $\left.\bar{P}_{i-1}\right)$. One computes the connecting homomorphism from $\operatorname{ker}\left(\bar{P}_{q+1}^{\prime \prime} / \bar{B}_{q+1}^{\prime \prime} \rightarrow \bar{Z}_{q}^{\prime \prime}\right)$ to $\operatorname{coker}\left(\bar{P}_{q+1}^{\prime} / \bar{B}_{q+1}^{\prime} \rightarrow \bar{Z}_{q}^{\prime}\right)$ by a diagram chase, which accounts in taking preimages twice, 
namely under the maps $\bar{P}_{q+1} / \bar{B}_{q+1} \rightarrow \bar{P}_{q+1}^{\prime \prime} / \bar{B}_{q+1}^{\prime \prime}$ and $\bar{Z}_{q}^{\prime} \rightarrow \bar{Z}_{q}$. For this the procedure Preimage from 3.1.4 is used.

Applying a contravariant functor to $(P)$ yields a long exact cohomology sequence. The corresponding procedure is called LongExactCohomologySeq.

\section{Appendix A. The RING PACKAges}

The following Maple ring packages have successfully been used with homalg. In each of the following descriptions we append a list of rings which can be dealt with in homalg using the respective package. Further, and without any extra help from the ring package, homalg can automatically compute over residue class rings of any supported ring.

- PIR [Bar07] is one more tiny package, or rather a pseudo-package, that makes Maple's built-in facilities for dealing with integers and some other principal ideal rings available to homalg. (Prime subfields $\mathbb{Q}$ and $\mathbb{Z} / p \mathbb{Z}$ and their finite field extensions, realized as primitive extensions, rational function fields over the previous fields, the integers $\mathbb{Z}$, the GAUssian integers $\mathbb{Z}[\sqrt{-1}]$ and univariate polynomial rings $\mathbb{Z} / p \mathbb{Z}[x]$, where $p$ is a prime, $\mathbb{Q}[x]$ and $K[x]$, where $K$ is a rational function field over a finite extension of $\mathbb{Q}$, realized as a primitive extension.)

- Involutive $\left[\mathrm{BCG}^{+} 03\right]$ implements the involutive basis technique of V. P. GERDT and Y. A. Blinkov in Maple. An involutive basis is a special kind of GröBner basis for an ideal of a polynomial ring or, more generally, for a submodule of a free module over a polynomial ring. Involutive bases have nice combinatorial properties [PR05], and the algorithms designed by V. P. GerdT and Y. A. Blinkov [Ger05, GB98a, GB98b] compute them efficiently. In fact, these algorithms provide an efficient alternative to BUCHBERGER's algorithm [Buc06] to compute GRÖBNER bases. Involutive restricts to particular involutive bases, namely JANET bases. It also provides an interface to a $\mathrm{C}++$ implementation of the involutive basis technique which can be used to call the fast routines when needed as well as to switch to these fast routines for the whole Maple session. (Commutative polynomial rings: $S\left[x_{1}, \ldots, x_{n}\right]$, where $S$ is either $\mathbb{Z}$ or a field existing in Maple.)

- Janet $\left[\mathrm{BCG}^{+} 03\right]$ implements the involutive basis technique for computing JANET bases of linear systems of partial differential equations. (Differential algebras over differential fields: $K\left[\frac{\partial}{\partial x_{1}}, \ldots, \frac{\partial}{\partial x_{n}}\right]$, where $K$ is a differential field which exists in Maple.)

- JanetOre [Rob06, Rob07] generalizes Involutive from commutative polynomial rings to certain iterated skew polynomial rings. In particular, it computes Janet bases for left ideals in Ore algebras [CS98]. ( $K[\partial ; \sigma, \delta]$, where $K$ is a polynomial ring over a field, $\partial$ a new indeterminate, $\sigma$ is a certain automorphism of $K$ and $\delta$ a $\sigma$-derivation of $K$, and iterated extensions of this kind.)

- OreModules [CQR07] is a Maple package for the study of structural properties of linear systems over ORE algebras, i.e. linear equations involving certain linear functional operators which can be considered as elements of an ORE algebra. By default, it uses the Maple package Ore_algebra [CS98] to compute GröBNER bases, 
but these calls can also be switched to JanetOre. (ORE algebras [CS98] and the iterated skew polynomial rings from the previous point.)

homalg is also able to make use of various normal form algorithms for modules resp. special types of modules over various rings, which are used to provide a standard form for a presentation of these modules:

- PIR uses the SMith normal form for (Maple-built-in) principal ideal rings.

- Janet optionally uses the JACOBSON normal form for univariate differential rings, i.e. rings of the form $K[\partial]$, where $K$ is a differential field with $\partial$ a derivation of $K$.

- Involutive optionally uses the extension package QuillenSuslin written by ANNA FABIANSKA [Fab07, FQ07] implementing the QUILLEN-SUSLIN theorem to compute a free basis of a projective module over a polynomial ring (which is then free by the theorem). A similar extension package is planned for OreModules.

- OreModules optionally uses the extension package Stafford [QR] which computes a free basis for a stably free module of rank at least 2 over the Weyl algebras $k\left[x_{1}, \ldots, x_{n}, \partial_{1}, \ldots, \partial_{n}\right]$ and $k\left(x_{1}, \ldots, x_{n}\right)\left[\partial_{1}, \ldots, \partial_{n}\right]$, with $k$ a field of characteristic 0 .

\section{Appendix B. The Maple implementation}

B.1. Presentations. A presentation of a module in the current Maple implementation of homalg is a list ${ }^{19}$ containing as first entry the list of generators and as second entry the list of relations. The third entry is a string delimiter to optically indicate the end of the presentation. This string, unless changed by the user, defaults to "Presentation". The remaining entries provide extra information about the presented module, e.g. its HILBERT series in case the ring is the polynomial ring. This extra information can only be provided by the ring-specific package.

For $M=\operatorname{coker}(\mathrm{M})=R^{1 \times l_{0}} / R^{1 \times l_{1}} \mathrm{M}$ the list of the concrete generators are numbered by abstract generators being the $l_{0}$ standard basis row vectors of the underlying free module $R^{1 \times l_{0}}$. The list of relations simply contains the rows of the matrix M. An illustration is given in Figure 1 of Example C.1.

B.2. The morphism part of functors. The name convention for functors used in the Maple implementation of homalg is as follows: If the procedure implementing the object part of a functor is called $\mathrm{F}$, then the procedure implementing the morphism part is called FMap. It is defined using the procedure FunctorMap applied to the object part procedure F. The several pieces of code for the morphism part of the functors Cokernel, Kernel, DefectOfHoms and Hom_R, and the two bifunctors TensorProduct and Hom, reproduced on the web [BR07a], demonstrate how FunctorMap unifies the definition of the morphism part of all functors in homalg.

In future implementations of homalg the two procedures implementing the object and morphism part of a functor will be unified in one. The unified procedure will be able to

\footnotetext{
${ }^{19}$ In future implementations we will make use of object oriented data structures, which encapsulate all this information.
} 
recognize if it has been applied to an object, to a morphism or even to complexes. This will be an easy task, once we strictly use structures throughout homalg.

B.3. Encapsulating functors. A functor is fully defined when both parts are defined, i.e. its object part and its morphism part. If the functor is a multi-functor, then several morphism parts have to be defined. homalg accesses all these parts of a functor via a so called encapsulation. It incorporates both parts and also takes care of the possible multi-functoriality. The implementation of the encapsulations of the functors Hom $R$, TensorProduct and Hom can be viewed under [BR07a].

B.4. Composition of functors. As was mentioned in Section 5, composing two functors is an easy task since one simply has to compose their actions on objects and on morphisms. The procedure responsible for this is called ComposeFunctors. The implementation of the composition of the functors HomHom_R and HomHom can be viewed under [BR07a].

B.5. Applying functors to complexes. As mentioned in Section 7, functors should be applied to complexes of modules, rather than to single modules. Thereby homalg again makes use of the encapsulation of functors. In this implementation, there are two procedures depending on whether one is dealing with a covariant or a contravariant functor. They are called FunctorOnSeqs and CofunctorOnSeqs. The implementation of the functors Hom and HomHom on complexes can be viewed under [BR07a].

B.6. Derivation of functors. The left derivation procedure for covariant functors is called LeftDerivedFunctor. The faster derivation procedure for right exact functors, for example $-\otimes L$, referred to at the end of Subsection 7.1 is called LeftDerivedRightExactFunctor.

The right derivation procedure for contravariant functors is called RightDerivedCofunctor. The faster derivation procedure for left exact contravariant functors, for example $\operatorname{Hom}(-, L)$, referred to at the end of Subsection 7.1 is called RightDerivedLeftExactCofunctor.

The implementation of the left derived functor LHomHom (of HomHom with respect to its first argument) and the right derived functor Ext (of Hom with respect to its first argument) can be viewed under [BR07a].

B.7. How FunctorMap works. Let $\mathrm{F}$ denote the object part procedure of a functor $F$. First FunctorMap asks $\mathrm{F}$ if the underlying functor is co- or contravariant. If $\mathrm{F}$ is homalgbasic it has to know the answer itself. If $\mathrm{F}$ is defined as a composition $F_{1} \circ F_{2}$ then the question is passed to the procedure ComposeFunctors, which decides the answer by asking $F_{1}$ and $F_{2}$ (this is recursive). If $\mathrm{F}$ is defined as a derivation $\mathrm{L}_{q} G$ or $\mathrm{R}^{q} G$, using one of the derivation procedures, then $\mathrm{F}$ passes the question to the latter, which decides the answer by asking $G$ (this is recursive). Any recursion ends when a homalg-basic functor is reached.

FunctorMap then asks $\mathrm{F}$ if it is defined using the procedure ComposeFunctors. If $\mathrm{F}$ is homalg-basic it ignores the question. If $\mathrm{F}$ is defined using one of the derivation procedures, the question is passed to the latter, which ignores it. If $\mathrm{F}$ is indeed defined as a composition $F_{1} \circ F_{2}$, then $\mathrm{F}$ passes the question to ComposeFunctors, which returns the two functors $F_{1}$ 
and $F_{2}$ in both their parts. FunctorMap can now easily construct the morphism part of $F$ by composing the morphism parts of $F_{1}$ and $F_{2}$. In case $\mathrm{F}$ is not defined as a composition, FunctorMap asks it if is defined by derivation. If $\mathrm{F}$ is homalg-basic it ignores the question. If $\mathrm{F}$ is indeed defined as a derivation $\mathrm{L}_{q} G$ or $\mathrm{R}^{q} G$, using one of the derivation procedures, then $\mathrm{F}$ passes the question to the latter, which returns the functor $G$ in both its parts and a procedure based on ResolutionOfSeq to compute $\varphi_{q}$ out of $\varphi$ (cf. (Lift), p. 18). With these two ingredients FunctorMap is able to construct the morphism part of $\mathrm{F}$ as described in Subsection 7.2. If $\mathrm{F}$ is defined neither by composition nor by derivation, i.e. its is homalg-

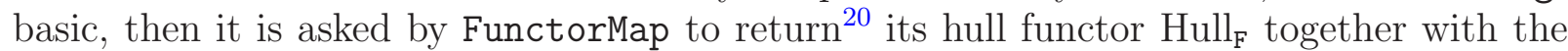
natural embedding (cf. (Hull), p. 10). Again this suffices to construct the morphism part of $\mathrm{F}$ as described in Section 5.

\section{Appendix C. Examples}

For further examples we refer to [BR06a, BR06b] and the site of homalg [BR07c].

A nice application is the tiny homalg-based package conley that computes $C$-connection matrices of graded module octahedra/braids of Morse decompositions in dynamical system theory $[\mathrm{BR}]$.

For the sake of demonstration we wrote a tiny package called alexander [BR07b], which relies on homalg and computes simplicial homology and cohomology. Future implementations of homalg are planned to enable more serious applications to topology.

C.1. Example 1. In this example we compute a module of homomorphisms.

$>$ restart:

$>$ with(Involutive): with(homalg):

Specify the homalg-table of the ring package Involutive:

> RPI:='Involutive/homalg';

$$
R P I:=\text { Involutive/homalg }
$$

Use the ring package Involutive as the default ring package:

$>$ 'homalg/default': =RPI;

$$
\text { homalg/default }:=\text { Involutive/homalg }
$$

Define the $\operatorname{ring} R=\mathbb{Q}[x, y, z]$ :

$>\operatorname{var}:=[\mathrm{x}, \mathrm{y}, \mathrm{z}]$;

$$
\begin{gathered}
\operatorname{var}:=[x, y, z] \\
\left.>\mathrm{K}:=\text { Cokernel }\left([\mathrm{x}, \mathrm{y}, 0],\left[\mathrm{x}^{\wedge} 2, \mathrm{y}^{\wedge} 2,0\right],\left[\mathrm{x} \wedge 3, \mathrm{y} \wedge 3, \mathrm{z}^{\wedge} 3\right]\right], \mathrm{var}\right) \\
K:=[[[1,0,0]=[1,0,0],[0,1,0]=[0,1,0],[0,0,1]=[0,0,1]], \\
{\left[[x, y, 0],\left[0, x y-y^{2}, 0\right],\left[0,0, z^{3}\right]\right], \text { "Presentation", }} \\
\left.3+8 s+14 s^{2}+s^{3}\left(\frac{14}{1-s}+\frac{6}{(1-s)^{2}}\right),[14,6,0]\right]
\end{gathered}
$$

\footnotetext{
${ }^{20}$ The technical details to realize this heavily depend on the implementation.
} 
Figure 1. A module of homomorphisms between two modules over $R=$ $\mathbb{Q}[x, y, z]$ with Involutive

$$
\begin{aligned}
& {\left[\left[[1,0,0]=\left[\begin{array}{lll}
1 & 0 & 0 \\
0 & 1 & 0
\end{array}\right],[0,1,0]=\left[\begin{array}{ccc}
0 & 0 & -y \\
0 & 0 & x
\end{array}\right],[0,0,1]=\left[\begin{array}{ccc}
0 & 0 & 0 \\
0 & x-y & 0
\end{array}\right]\right],\right.} \\
& \mid \begin{array}{c}
{\left[[0,0, y],\left[x^{2}-x y, 0,-x\right],\left[0, z^{3}, 0\right]\right],} \\
\text { generators } \\
\text { "Presentation", } \\
3+8 s+14 s^{2}+s^{3}\left(\frac{14}{(1-s)}+\frac{6}{(1-s)^{2}}\right),
\end{array} \\
& \text { relations } \\
& \text { HilberT series }
\end{aligned}
$$

$>\quad \mathrm{L}:=\operatorname{Cokernel}([\mathrm{x}, \mathrm{y}]], \operatorname{var})$;

$$
\begin{aligned}
& L:=[[[1,0]=[1,0],[0,1]=[0,1]],[[x, y]], \text { "Presentation", } \\
& \left.2+s\left(\frac{2}{1-s}+\frac{2}{(1-s)^{2}}+\frac{1}{(1-s)^{3}}\right),[2,2,1]\right]
\end{aligned}
$$

Compute the module of homomorphisms $\operatorname{Hom}_{R}(L, K)$ (see Figure 1):

$>\operatorname{hom}:=\operatorname{Hom}(\mathrm{L}, \mathrm{K}, \mathrm{var})$;

$$
\begin{aligned}
& \text { hom }:=\left[\left[[1,0,0]=\left[\begin{array}{lll}
1 & 0 & 0 \\
0 & 1 & 0
\end{array}\right],[0,1,0]=\left[\begin{array}{ccc}
0 & 0 & -y \\
0 & 0 & x
\end{array}\right],[0,0,1]=\left[\begin{array}{ccc}
0 & 0 & 0 \\
0 & -y+x & 0
\end{array}\right]\right.\right. \\
& {\left[[0,0, y],\left[x^{2}-x y, 0,-x\right],\left[0, z^{3}, 0\right]\right], \text { "Presentation", }} \\
& \left.3+8 s+14 s^{2}+s^{3}\left(\frac{14}{1-s}+\frac{6}{(1-s)^{2}}\right),[14,6,0]\right]
\end{aligned}
$$

C.2. Example 2. Let $R:=\mathbb{Q}[x, y, z]$. In this example we want to study two nonequivalent extensions $0 \rightarrow K \rightarrow M \rightarrow L \rightarrow 0$ and $0 \rightarrow K \rightarrow N \rightarrow L \rightarrow 0$ with $K$ a torsion module and $L$ a torsion free module. Our goal is to use the notion of functor to reveal that these extensions are not only non-equivalent but also non-isomorphic. For this we define two functors $F_{M}$ and $F_{N}$ and study their behavior when applied to complexes. Concretely, we apply $F_{M}$ resp. $F_{N}$ to $0 \rightarrow K \rightarrow M \rightarrow L \rightarrow 0$ which we refer to as

$$
S: 0 \rightarrow K \stackrel{\alpha_{1}}{\rightarrow} M \stackrel{\alpha_{2}}{\rightarrow} L \rightarrow 0 .
$$

In the following we consider

$$
F_{Q}=\operatorname{Ext}(1,-, Q)=\operatorname{Ext}^{1}(-, Q)=R^{1} \operatorname{Hom}_{R}(-, Q)
$$

$>$ restart;

$>$ with(Involutive): with(homalg):

Use the ring package Involutive as the default ring package: 
> RPI:='Involutive/homalg';

$$
R P I:=\text { Involutive/homalg }
$$

$>$ 'homalg/default': =RPI;

$$
\text { homalg/default }:=\text { Involutive/homalg }
$$

We force the Maple ring package Involutive to use the external program JB, which is a $\mathrm{C}++$ implementation of the involutive division algorithm:

$>$ InvolutiveOptions("C++");

Define the ring $R=\mathbb{Q}[x, y, z]$ :

$>\operatorname{var}:=[\mathrm{x}, \mathrm{y}, \mathrm{z}]$

$$
\operatorname{var}:=[x, y, z]
$$

The two presentation matrices $\mathrm{M}$ and $\mathrm{N}$ :

$>$ MM:=matrix $\left(\left[\left[x * z, z * y, z^{\wedge} 2,0,0, y\right],\left[0,0,0, z^{\wedge} 2 * y-z \wedge 2, z^{\wedge} 3, x * z\right]\right.\right.$,

$>\left[0,0,0, z^{*} * y^{\wedge} 2-z * y, z^{\wedge} 2 * y, x * y\right],\left[0,0,0, x * z * y-x * z, x * z^{\wedge} 2, x^{\wedge} 2\right]$,

$>\left[-\mathrm{x} * \mathrm{y},-\mathrm{y}^{\wedge} 2,-\mathrm{z} * \mathrm{y}, \mathrm{x}^{\wedge} 2 * \mathrm{y}-\mathrm{x}^{\wedge} 2-\mathrm{y}+1, \mathrm{x}^{\wedge} 2 * \mathrm{z}-\mathrm{z}, 0\right],\left[\mathrm{x}^{\wedge} 2 * \mathrm{y}-\mathrm{x}^{\wedge} 2, \mathrm{x}^{*} * \mathrm{y}^{\wedge} 2-\mathrm{x} * \mathrm{y}\right.$, $\left.\left.\left.>\quad \mathrm{x} * \mathrm{z} * \mathrm{y}-\mathrm{x} * \mathrm{z}, \quad-\mathrm{y}^{\wedge} 3+2 * \mathrm{y}^{\wedge} 2-\mathrm{y},-\mathrm{z}^{*} \mathrm{y}^{\wedge} 2+\mathrm{z} * \mathrm{y}, 0\right]\right]\right)$;

$M M:=\left[\begin{array}{cccccc}x z & z y & z^{2} & 0 & 0 & y \\ 0 & 0 & 0 & z^{2} y-z^{2} & z^{3} & x z \\ 0 & 0 & 0 & z y^{2}-z y & z^{2} y & x y \\ 0 & 0 & 0 & x z y-x z & x z^{2} & x^{2} \\ -x y & -y^{2} & -z y & x^{2} y-x^{2}-y+1 & x^{2} z-z & 0 \\ x^{2} y-x^{2} & x y^{2}-x y & x z y-x z & -y^{3}+2 y^{2}-y & -z y^{2}+z y & 0\end{array}\right]$

$>N N:=\operatorname{matrix}\left(\left[\left[x * y, y^{\wedge} 2, z * y, 0,0, z\right],\left[0,0,0, z * y^{\wedge} 2-z * y, z^{\wedge} 2 * y\right.\right.\right.$, $>\mathrm{x} * \mathrm{z}],\left[\mathrm{x}^{\wedge} 2 * \mathrm{z}, \mathrm{x} * \mathrm{z} * \mathrm{y}, \mathrm{x} * \mathrm{z}^{\wedge} 2,-\mathrm{z}^{\wedge} 2 * \mathrm{y}+\mathrm{z}^{\wedge} 2,-\mathrm{z}^{\wedge} 3,0\right],[0,0,0$,

$\left.>\quad y^{\wedge} 3-2 * y^{\wedge} 2+y, z * y^{\wedge} 2-z * y, x * y-x\right],\left[0,0,0, x^{\wedge} 2 * y-x^{\wedge} 2-y+1, x \wedge 2 * z-z, y\right]$, $\left.\left.>\left[\mathrm{x}^{\wedge} 3, \mathrm{x}^{\wedge} 2 * \mathrm{y}, \mathrm{x}^{\wedge} 2 * \mathrm{z},-\mathrm{x} * \mathrm{z} * \mathrm{y}+\mathrm{x} * \mathrm{z},-\mathrm{x} * \mathrm{z}^{\wedge} 2,0\right]\right]\right)$;

$$
N N:=\left[\begin{array}{cccccc}
x y & y^{2} & z y & 0 & 0 & z \\
0 & 0 & 0 & z y^{2}-z y & z^{2} y & x z \\
x^{2} z & x z y & x z^{2} & -z^{2} y+z^{2} & -z^{3} & 0 \\
0 & 0 & 0 & y^{3}-2 y^{2}+y & z y^{2}-z y & x y-x \\
0 & 0 & 0 & x^{2} y-x^{2}-y+1 & x^{2} z-z & y \\
x^{3} & x^{2} y & x^{2} z & -x z y+x z & -x z^{2} & 0
\end{array}\right]
$$

The two modules $M:=\operatorname{coker}\left(R^{1 \times 6} \stackrel{\mathrm{M}}{\rightarrow} R^{1 \times 6}\right)$ and $N:=\operatorname{coker}\left(R^{1 \times 6} \stackrel{\mathrm{N}}{\rightarrow} R^{1 \times 6}\right)$ :

$>\mathrm{M}:=$ Cokernel $(\mathrm{MM}, \mathrm{var})$; 
$M:=[[[1,0,0,0,0,0]=[1,0,0,0,0,0],[0,1,0,0,0,0]=[0,1,0,0,0,0]$,

$[0,0,1,0,0,0]=[0,0,1,0,0,0],[0,0,0,1,0,0]=[0,0,0,1,0,0]$,

$[0,0,0,0,1,0]=[0,0,0,0,1,0],[0,0,0,0,0,1]=[0,0,0,0,0,1]],[$

$\left[x z, z y, z^{2}, 0,0, y\right],\left[0,0,0, z^{2} y-z^{2}, z^{3}, x z\right],\left[0,0,0, z y^{2}-z y, z^{2} y, x y\right]$,

$\left[0,0,0, x z y-x z, x z^{2}, x^{2}\right],\left[x^{2} z, x z y, x z^{2}, 0,0, x y\right]$

$\left[-x y,-y^{2},-z y, x^{2} y-x^{2}-y+1, x^{2} z-z, 0\right]$

$\left[x^{2} y-x^{2}, x y^{2}-x y, x z y-x z,-y^{3}+2 y^{2}-y,-z y^{2}+z y, 0\right]$,

$\left.\left[0,0,0, z y-z, z^{2}, x^{3}-y^{2}\right]\right]$, "Presentation",

$\frac{3 s^{2}}{1-s}+\frac{2 s}{1-s}+\frac{2}{(1-s)^{2}}+\frac{3}{(1-s)^{3}}+\frac{2 s^{2}}{(1-s)^{2}}+s^{2}+s+\frac{1}{1-s}+\frac{s}{(1-s)^{2}}$,

$[34,14,3]]$

$>\mathrm{N}:=\operatorname{Cokernel}(\mathrm{NN}, \mathrm{var})$;

$N:=[[[1,0,0,0,0,0]=[1,0,0,0,0,0],[0,1,0,0,0,0]=[0,1,0,0,0,0]$,

$[0,0,1,0,0,0]=[0,0,1,0,0,0],[0,0,0,1,0,0]=[0,0,0,1,0,0]$,

$[0,0,0,0,1,0]=[0,0,0,0,1,0],[0,0,0,0,0,1]=[0,0,0,0,0,1]],[$

$\left[x y, y^{2}, z y, 0,0, z\right],\left[0,0,0, z y^{2}-z y, z^{2} y, x z\right],\left[x^{2} z, x z y, x z^{2},-z^{2} y+z^{2},-z^{3}, 0\right]$,

$\left[0,0,0, y^{3}-2 y^{2}+y, z y^{2}-z y, x y-x\right],\left[0,0,0, x^{2} y-x^{2}-y+1, x^{2} z-z, y\right]$,

$\left[x^{2} y, x y^{2}, x z y, 0,0, x z\right],\left[x^{3}, x^{2} y, x^{2} z,-x z y+x z,-x z^{2}, 0\right]$

$\left[0,0,0, x y^{2} z-x z y, x y z^{2}, x^{2} z\right],\left[0,0,0,0,0, x^{3} z-z y^{2}-x z\right]$

$\left[0,0,0, x y^{3}-2 x y^{2}+x y, x y^{2} z-x z y, x^{2} y-x^{2}\right]$,

$\left.\left[0,0,0,0,0, x^{3} y-x^{3}-y^{3}-x y+y^{2}+x\right]\right]$, "Presentation", $\frac{3 s}{1-s}+\frac{2}{(1-s)^{2}}+2 s^{2}$

$\left.+\frac{3}{(1-s)^{3}}+\frac{2 s^{2}}{(1-s)^{2}}+\frac{s^{2}}{1-s}+s^{3}+\frac{1}{1-s}+\frac{s^{3}}{1-s}+\frac{s}{(1-s)^{2}},[51,17,3]\right]$

The torsion submodule $K:=t(M)$ of $M($ and $N)$ :

$>\mathrm{K}:=$ TorsionSubmodule $(\mathrm{M}, \mathrm{var})$;

$K:=[[[1,0,0]=[0,0,0,0,0,1],[0,1,0]=[0,0,0, y-1, z, 0],[0,0,1]=[x, y, z, 0,0,0]$ ],$\left[[y, 0, z],[0,-z y, x z],\left[x z, z^{2}, 0\right],\left[0,-y^{2}+y, x y-x\right],[x y, z y, 0],\left[0, x^{2}-1,-y\right]\right.$, $\left.\left[x^{2}, x z, 0\right]\right]$, "Presentation", $\left.s+\frac{1}{1-s}+\frac{s}{(1-s)^{2}}+\frac{2}{(1-s)^{2}}+\frac{s}{1-s},[7,3,0]\right]$

The embedding $0 \rightarrow K \stackrel{\alpha_{1}}{\rightarrow} M$ :

> alpha1:=TorsionSubmoduleEmb(M, var); 


$$
\alpha 1:=\left[\begin{array}{cccccc}
0 & 0 & 0 & 0 & 0 & 1 \\
0 & 0 & 0 & y-1 & z & 0 \\
x & y & z & 0 & 0 & 0
\end{array}\right]
$$

The torsion free factor $L:=M / K=M / t(M)$ of $M($ and $N)$ :

$>\mathrm{L}:=$ Cokernel (alpha1, M, var);

$$
\begin{aligned}
& L:=[[[1,0,0,0,0]=[1,0,0,0,0,0],[0,1,0,0,0]=[0,1,0,0,0,0], \\
& {[0,0,1,0,0]=[0,0,1,0,0,0],[0,0,0,1,0]=[0,0,0,1,0,0],} \\
& [0,0,0,0,1]=[0,0,0,0,1,0]],[[0,0,0, y-1, z],[x, y, z, 0,0]], \text { "Presentation", } \\
& \left.\frac{2}{(1-s)^{2}}+\frac{3}{(1-s)^{3}},[5,5,3]\right]
\end{aligned}
$$

The natural epimorphism $M \stackrel{\alpha_{2}}{\longrightarrow} L \rightarrow 0$ :

> alpha2:=CokernelEpi (alpha1,M,var);

$$
\alpha 2:=\left[\begin{array}{ccccc}
1 & 0 & 0 & 0 & 0 \\
0 & 1 & 0 & 0 & 0 \\
0 & 0 & 1 & 0 & 0 \\
0 & 0 & 0 & 1 & 0 \\
0 & 0 & 0 & 0 & 1 \\
0 & 0 & 0 & 0 & 0
\end{array}\right]
$$

The short sequence $S: 0 \rightarrow K \stackrel{\alpha_{1}}{\rightarrow} M \stackrel{\alpha_{2}}{\rightarrow} L \rightarrow 0$ is exact:

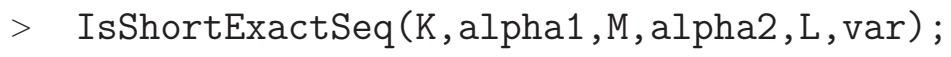

true

The functor $\operatorname{Ext}_{R}^{1}(-, N)$ applied to the short exact sequence $S$ :

$>\operatorname{coseqN}:=\operatorname{ExtOnSeqs}(1, \mathrm{~N},[\mathrm{alpha}$, alpha1], $[\mathrm{L}, \mathrm{M}, \mathrm{K}], \mathrm{var})$ :

This says that the map $\operatorname{Ext}_{R}^{1}(L, N) \stackrel{\operatorname{Ext}_{R}^{1}\left(\alpha_{2}, N\right)}{\longrightarrow} \operatorname{Ext}_{R}^{1}(M, N)$ is not injective, i.e. that the connecting homomorphism $\operatorname{Hom}_{R}(K, N) \stackrel{\delta^{0}}{\rightarrow} \operatorname{Ext}_{R}^{1}(L, N)$ is non-trivial:

$>$ IsShortExactSeq (op (MakeCoseq (coseqN)), var, "VERBOSE");

"homs" = true, "cmps" = true, "defs" =

$$
\left[\left[\left[1=\left[\begin{array}{llllll}
0 & 0 & 0 & 0 & 0 & 0 \\
0 & 0 & 0 & 0 & 0 & 1
\end{array}\right]\right],[z, y, x], \text { "Presentation", } 1,[0,0,0]\right] \text {, true, true }\right]
$$

The functor $\operatorname{Ext}_{R}^{1}(-, M)$ applied to the short exact sequence $S$ :

$>\operatorname{coseqM}:=\operatorname{ExtOnSeqs}(1, \mathrm{M},[\mathrm{alpha} 2$, alpha1], [L, M, K], var) :

Whereas the resulting sequence $\operatorname{Ext}_{R}^{1}(S, M)$ is again exact. In particular, the connecting homomorphism $\operatorname{Hom}_{R}(K, M) \stackrel{\delta^{0}}{\rightarrow} \operatorname{Ext}_{R}^{1}(L, M)$ is trivial, i.e. the zero map:

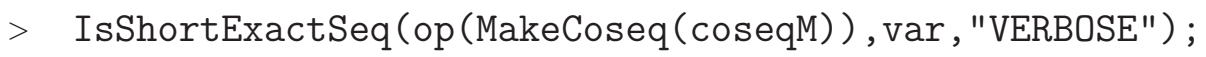


C.3. Example 3. This example demonstrates by a computation over a noncommutative OrE domain how one can use homalg to solve a linear system of differential-difference equations:

$>$ restart:

$>$ with(JanetOre): with(homalg):

Specify the homalg-table of the ring package JanetOre:

$>$ RPO:='JanetOre/homalg';

$$
R P O:=\text { JanetOre/homalg }
$$

Use the ring package JanetOre as the default ring package:

$>$ 'homalg/default': =RPO;

$$
\text { homalg/default }:=\text { JanetOre/homalg }
$$

Ask homalg to check if a stably free module with a finite free resolution ${ }^{21}$ is free, cf. [QR, Remark 51]:

$>$ homalg_options ("check_rank_1"=true);

"JanetOre/homalg", "check_if_stably_free_rank_1_FFR_is_free" = true

Define the Ore domain $R=\mathbb{Q}[t]\left[D, t \mapsto t, \frac{d}{d t}\right][\delta, t \mapsto t+1,0]$ :

$>$ Ore: $=[[t, D, \operatorname{delta}],[],[\operatorname{weyl}(D, t), \operatorname{shift}(\operatorname{delta}, t)]]$;

$$
\text { Ore }:=[[t, \mathrm{D}, \delta],[],[\operatorname{weyl}(\mathrm{D}, t), \operatorname{shift}(\delta, t)]]
$$

Find the general solution to the following linear system of differential-difference equations:

$$
\begin{aligned}
u(t)+u(t+2)+\mathrm{D}(v)(t+1)-v(t+2)+w(t+1) & =0, \\
t \mathrm{D}(u)(t+1)+u(t+3)-2 t u(t+1)+2 \mathrm{D}(u)(t+1)-2 u(t+1) & \\
-v(t+3)+2 v(t+2)+w(t+2) & =0 .
\end{aligned}
$$

This system can be written as the following matrix operators $A$ applied to the section $\left(\begin{array}{lll}u(t) & v(t) & w(t)\end{array}\right)^{\mathrm{tr}}$. (Caution: A monomial in $t, D$ and $\delta$ must be read as a monomial with powers of $t$ on the left of a monomial in the commuting $D$ and $\delta$ : read in the following both $t D, D t$ as $t D$, and both $t \delta, \delta t$ as $t \delta)$ :

$>A:=\operatorname{matrix}\left(\left[\left[1+\operatorname{delta}{ }^{\wedge} 2\right.\right.\right.$, delta $* \mathrm{D}-\operatorname{delta}{ }^{\wedge} 2$, delta $]$,

$>[\mathrm{t} * \operatorname{delta} * \mathrm{D}+\operatorname{delta}$ 3-2*t*delta+2*delta*D-2*delta,$-\operatorname{delta} 3+2 * \operatorname{delta} 2$,

$>$ delta`2]]);

$$
A:=\left[\begin{array}{ccc}
1+\delta^{2} & \delta \mathrm{D}-\delta^{2} & \delta \\
t \delta \mathrm{D}+\delta^{3}-2 t \delta+2 \delta \mathrm{D}-2 \delta & -\delta^{3}+2 \delta^{2} & \delta^{2}
\end{array}\right]
$$

Studying the system is equivalent to studying the cokernel $M$ of $A \in R^{m \times n}$ viewed as a morphism $R^{1 \times m} \stackrel{A}{\rightarrow} R^{1 \times n}$. This is based on the following observation: Let $\mathcal{F}$ be a function space, where one wants to search for solutions of the system, then $\operatorname{Hom}_{R}(\operatorname{coker}(A), \mathcal{F}) \cong$ $\operatorname{Sol}_{\mathcal{F}}(A):=\left\{\eta \in \mathcal{F}^{n \times 1} \mid A \eta=0\right\}$, where $n$ is the number of columns of $A$.

\footnotetext{
${ }^{21}$ Without a finite free resolution homalg cannot check stably freeness, cf. [QR, Remark 28].
} 
$>\quad M:=\operatorname{Cokernel}(\mathrm{A}$, Ore $)$;

$$
\begin{aligned}
& M:=[[[1,0,0]=[1,0,0],[0,1,0]=[0,1,0],[0,0,1]=[0,0,1]], \\
& {\left[\left[1+\delta^{2}, \delta \mathrm{D}-\delta^{2}, \delta\right],\left[t \delta \mathrm{D}+\delta^{3}-2 t \delta+2 \delta \mathrm{D}-2 \delta,-\delta^{3}+2 \delta^{2}, \delta^{2}\right]\right],} \\
& \text { "Presentation", } \left.3+9 s+17 s^{2}+s^{3}\left(\frac{17}{1-s}+\frac{8}{(1-s)^{2}}+\frac{1}{(1-s)^{3}}\right),[17,8,1]\right]
\end{aligned}
$$

The torsion free factor $F$ of $M$ turns out to be free of rank 1 (the above mentioned option "check_rank_1" was used):

$>\mathrm{F}:=$ TorsionFreeFactor (M,Ore);

$$
F:=\left[[1=[-\delta, \delta-\mathrm{D},-1]],[0], \text { "Presentation", } \frac{1}{(1-s)^{3}},[0,0,1]\right]
$$

The natural epimorphsim $M \stackrel{\nu}{\rightarrow} F$ :

$>$ nu:=TorsionFreeFactorEpi(M,Ore);

$$
\nu:=\left[\begin{array}{c}
\delta \\
t \\
-2+\delta+t \delta-t \mathrm{D}-\delta^{2}
\end{array}\right]
$$

Since $F$ is free one can use Leftinverse to compute a split $M \stackrel{\chi}{\rightarrow} F$ (cf. 3.1.3,(1)):

$>$ chi:=Leftinverse (M, nu, F,Ore);

$$
\chi:=\left[\begin{array}{lll}
-\delta & \delta-\mathrm{D} & -1
\end{array}\right]
$$

Now compute the torsion submodule $T$ of $M$ :

$>\mathrm{T}:=$ TorsionSubmodule(M,Ore);

$$
\begin{aligned}
& T:=\left[\left[[1,0]=[t+1,-\delta, 0],[0,1]=\left[-2 \delta,-t \delta+t \mathrm{D}+\delta^{2}+1, t\right]\right],\right. \\
& {\left[\left[1+\delta^{2}, \delta\right],[-2+\mathrm{D},-2 \delta+\delta \mathrm{D}],[-2 \delta+\delta \mathrm{D}, 0]\right], \text { "Presentation", }} \\
& \left.2+6 s+s^{2}\left(\frac{6}{1-s}+\frac{3}{(1-s)^{2}}\right),[6,3,0]\right]
\end{aligned}
$$

And the natural embedding $T \stackrel{\iota}{\rightarrow} M$ :

$>$ iota:=TorsionSubmoduleEmb(M,Ore);

After a few tests we found an element $\mu \in T$

$$
\iota:=\left[\begin{array}{ccc}
t+1 & -\delta & 0 \\
-2 \delta & -t \delta+t \mathrm{D}+\delta^{2}+1 & t
\end{array}\right]
$$

$>\mathrm{mu}:=[[\operatorname{delta}, 1]]$;

$$
\mu:=[[\delta, 1]]
$$

which turned out to be a cyclic generator of $T$ (below one identifies $\mu$ with the morphism $\left.R^{1 \times 1} \rightarrow T: 1 \mapsto \mu\right):$

$>$ IsSurjective(mu, T,Ore); 
$H$ is nothing but $T$ rewritten on this cyclic generator. It further turns out that the cyclic generator $\mu$ of the torsion submodule $T$ satisfies a single simple relation $\delta^{2}(D-2) \mu=0$ :

$>\mathrm{H}:=\operatorname{Image}(\mathrm{mu}, \mathrm{T}, \mathrm{Ore})$;

$$
\begin{aligned}
& H:=\left[[1=[t \delta, t \mathrm{D}-t \delta+1, t]],\left[-2 \delta^{2}+\delta^{2} \mathrm{D}\right],\right. \text { "Presentation", } \\
& \left.1+3 s+6 s^{2}+s^{3}\left(\frac{6}{1-s}+\frac{3}{(1-s)^{2}}\right),[6,3,0]\right]
\end{aligned}
$$

$\varepsilon$ is nothing but $\iota$ rewritten on the cyclic generator $\mu$ :

> epsilon:=ComposeMaps (mu, iota, M,Ore);

$$
\varepsilon:=\left[\begin{array}{lll}
t \delta & t \mathrm{D}-t \delta+1 & t
\end{array}\right]
$$

$>\operatorname{IsHom}(\mathrm{H}$, epsilon, M,Ore);

$$
\text { true }
$$

$>$ IsInjective(H, epsilon, M,Ore);

true

Now construct the isomorphism $\alpha$ from the direct sum $S:=F \oplus H$ onto $M$ :

$>$ alpha:=RPO[matrix] (RPO[UnionOfRows] (chi,epsilon));

$>\mathrm{S}:=\operatorname{DirectSum}(\mathrm{F}, \mathrm{H}, \mathrm{Ore})$;

$$
\alpha:=\left[\begin{array}{ccc}
-\delta & \delta-\mathrm{D} & -1 \\
t \delta & t \mathrm{D}-t \delta+1 & t
\end{array}\right]
$$

$$
\begin{aligned}
& \quad S:=\left[[[1,0]=[1,0],[0,1]=[0,1]],\left[\left[0,-2 \delta^{2}+\delta^{2} \mathrm{D}\right]\right],\right. \text { "Presentation" } \\
& \left.\quad 2+6 s+12 s^{2}+s^{3}\left(\frac{12}{1-s}+\frac{6}{(1-s)^{2}}+\frac{1}{(1-s)^{3}}\right),[12,6,1]\right] \\
& >\quad \operatorname{IsHom(S,alpha,M,Ore);} \quad \text { true } \\
& >\text { IsBijective(S, alpha,M, Ore); }
\end{aligned}
$$

true

$N$ is now nothing but $M$ rewritten on the images of the two generators of $S$. So $M$ (or $N)$ is a module generated by two generators, one free and one subject to a single simple relation:

$>\quad \mathrm{N}:=$ Image (alpha, M,Ore);

$$
\begin{aligned}
& N:=\left[[[1,0]=[-\delta, \delta-\mathrm{D},-1],[0,1]=[t \delta, t \mathrm{D}-t \delta+1, t]],\left[\left[0,-2 \delta^{2}+\delta^{2} \mathrm{D}\right]\right],\right. \\
& \text { "Presentation", } \left.2+6 s+12 s^{2}+s^{3}\left(\frac{12}{1-s}+\frac{6}{(1-s)^{2}}+\frac{1}{(1-s)^{3}}\right),[12,6,1]\right]
\end{aligned}
$$

Using sections, the above presentation reads: The original system on the three unkown functions $u(t), v(t)$ and $w(t)$ is equivalent to a system on two unknown functions $f(t)$ and $g(t)$, where only $g(t)$ satisfies the single simple equation $D(g)(t+1)-g(t+1)=0$. It is also given how to express $f$ and $g$ in terms of $u, v$ and $w$. 
$>$ PresentationOnSections (N,Ore[1], Ore [3], [u,v,w], [f,g]);

$[[\mathrm{f}(t)=-\mathrm{u}(t+1)+\mathrm{v}(t+1)-\mathrm{D}(v)(t)-\mathrm{w}(t)$,

$\mathrm{g}(t)=t \mathrm{u}(t+1)+t \mathrm{D}(v)(t)-t \mathrm{v}(t+1)+\mathrm{v}(t)+t \mathrm{w}(t)],[-2 \mathrm{~g}(t+2)+\mathrm{D}(g)(t+2)]$,

"Presentation", $\left.2+6 s+12 s^{2}+s^{3}\left(\frac{12}{1-s}+\frac{6}{(1-s)^{2}}+\frac{1}{(1-s)^{3}}\right),[12,6,1]\right]$

$f(t)$ is therefore a free function and $g(t)=C \exp (t)$, where $C$ is an arbitrary constant:

$>$ sol:=[f=unapply $(f(t), t), g=\operatorname{unapply}(C * \exp (2 * t), t)]$;

$$
\text { sol }:=\left[f=(t \rightarrow \mathrm{f}(t)), g=\left(t \rightarrow C e^{(2 t)}\right)\right]
$$

In order to solve the original system one needs to express $u, v$ and $w$ in terms of $f$ and $g$. To this end use Leftinverse to compute $\eta:=\alpha^{-1}$ (cf. 3.1.3,(2)):

$>$ eta:=Leftinverse(S, alpha,M,Ore);

$$
\eta:=\left[\begin{array}{cc}
\delta & 0 \\
t & 1 \\
-2+\delta+t \delta-t \mathrm{D}-\delta^{2} & \delta-\mathrm{D}
\end{array}\right]
$$

Again, $L$ is nothing but $S(\cong M)$ rewritten on the images (under $\eta$ ) of the three original generators of $M$ :

$>\quad$ L:=Image (eta, S,Ore, "USE_IMAGE_OF_GENERATORS") ;

$$
\begin{aligned}
& L:=\left[\left[[1,0,0]=[\delta, 0],[0,1,0]=[t, 1],[0,0,1]=\left[-2+\delta+t \delta-t \mathrm{D}-\delta^{2}, \delta-\mathrm{D}\right]\right],\right. \\
& {\left[\left[1+\delta^{2}, \delta \mathrm{D}-\delta^{2}, \delta\right],\left[t \delta \mathrm{D}+\delta^{3}-2 t \delta+2 \delta \mathrm{D}-2 \delta,-\delta^{3}+2 \delta^{2}, \delta^{2}\right]\right],} \\
& \text { "Presentation", } \left.3+9 s+17 s^{2}+s^{3}\left(\frac{17}{1-s}+\frac{8}{(1-s)^{2}}+\frac{1}{(1-s)^{3}}\right),[17,8,1]\right]
\end{aligned}
$$

And again, using the JanetOre procedure PresentationOnSections, the above presentation is rewritten:

$>\quad \mathrm{P}:=$ PresentationOnSections (L,Ore [1], Ore [3], [f,g], [u, v, w] );

$$
\begin{aligned}
& P:=[[\mathrm{u}(t)=\mathrm{f}(t+1), \mathrm{v}(t)=t \mathrm{f}(t)+\mathrm{g}(t), \\
& \mathrm{w}(t)=-2 \mathrm{f}(t)+\mathrm{f}(t+1)+t \mathrm{f}(t+1)-t \mathrm{D}(f)(t)-\mathrm{f}(t+2)+\mathrm{g}(t+1)-\mathrm{D}(g)(t)],[ \\
& \mathrm{u}(t)+\mathrm{u}(t+2)+\mathrm{D}(v)(t+1)-\mathrm{v}(t+2)+\mathrm{w}(t+1), t \mathrm{D}(u)(t+1)+\mathrm{u}(t+3) \\
& -2 t \mathrm{u}(t+1)+2 \mathrm{D}(u)(t+1)-2 \mathrm{u}(t+1)-\mathrm{v}(t+3)+2 \mathrm{v}(t+2)+\mathrm{w}(t+2)], \\
& \text { "Presentation", } \left.3+9 s+17 s^{2}+s^{3}\left(\frac{17}{1-s}+\frac{8}{(1-s)^{2}}+\frac{1}{(1-s)^{3}}\right),[17,8,1]\right]
\end{aligned}
$$

The most general $C^{\infty}(\mathbb{R})$-solution is given by:

$>$ Sol:=eval (RPO[matrix] (GeneratorsOfPresentation(P)), sol);

$$
\text { Sol }:=\left[\begin{array}{c}
\mathrm{f}(t+1) \\
t \mathrm{f}(t)+C e^{(2 t)} \\
-2 \mathrm{f}(t)+\mathrm{f}(t+1)+t \mathrm{f}(t+1)-t \mathrm{D}(f)(t)-\mathrm{f}(t+2)+C e^{(2 t+2)}-2 C e^{(2 t)}
\end{array}\right]
$$


Besides, one has the following uniqueness property: If $\widetilde{f}$ and $\widetilde{C}$ lead to the same solution then $\widetilde{f}=f$ and $\widetilde{C}=C$.

Test the general solution with the JanetOre procedure JApplyMatrix:

> JApplyMatrix(A,Sol,Ore[1],Ore[3]);

$$
\left[\begin{array}{l}
0 \\
0
\end{array}\right]
$$

\section{REFERENCES}

[Bar07] Mohamed Barakat, PIR: A tiny homalg ring package for Maple-built-in principal ideal rings, 2004-2007, (http://wwwb.math.rwth-aachen. de:8040). 20

$\left[\mathrm{BCG}^{+} 03\right]$ Y. A. Blinkov, C. F. Cid, V. P. Gerdt, W. Plesken, and D. Robertz, The MAPLE Package JAnet: I. Polynomial Systems. II. Linear Partial Differential Equations, Proc. 6th Int. Workshop on Computer Algebra in Scientific Computing, Passau, 2003, (http://wwwb.math.rwth-aachen.de/Janet). 20

[BGTV03] José Bueso, José Gómez-Torrecillas, and Alain Verschoren, Algorithmic methods in noncommutative algebra, Mathematical Modelling: Theory and Applications, vol. 17, Kluwer Academic Publishers, Dordrecht, 2003, Applications to quantum groups. MR MR2006329 (2005c:16069) 4

[Bjö79] J.-E. Björk, Rings of differential operators, North-Holland Mathematical Library, vol. 21, North-Holland Publishing Co., Amsterdam, 1979. MR MR549189 (82g:32013) 2

[BR] Mohamed Barakat and Daniel Robertz, conley - computing connection matrices in Maple, Accepted for publication in the JSC. arXiv:math.DS/0701173 and (http://wwwb.math.rwth-aachen.de/conley). 23

[BR06a] Computing invariants of multidimensional linear systems on an abstract homological level, Proceedings of the 17th International Symposium on Mathematical Theory of Networks and Systems (MTNS 2006), Kyoto (Japan), 2006, pp. 542-559. 23

[BR06b] homalg: First steps to an abstract package for homological algebra, Proceedings of the $\mathrm{X}$ meeting on computational algebra and its applications (EACA 2006), Sevilla (Spain), 2006, pp. 29-32. 23

[BR07a] , homalg code, 2004-2007, (http://wwwb.math.rwth-aachen.de/homalg/implementation.html). 21,22

[BR07b] - alexander: A sample homalg-based Maple package to compute simplicial homology and cohomology, 2004-2007, (http://wwwb.math.rwth-aachen.de:8040). 23

[BR07c] , homalg project, 2004-2007, (http://wwwb.math.rwth-aachen.de/homalg). 5, 23

[Buc06] Bruno Buchberger, An algorithm for finding the basis elements of the residue class ring of a zero dimensional polynomial ideal, J. Symbolic Comput. 41 (2006), no. 3-4, 475-511, Translated from the 1965 German original by Michael P. Abramson. MR MR2202562 (2006m:68184) 20

[CE99] Henri Cartan and Samuel Eilenberg, Homological algebra, Princeton Landmarks in Mathematics, Princeton University Press, Princeton, NJ, 1999, With an appendix by David A. Buchsbaum, Reprint of the 1956 original. MR MR1731415 (2000h:18022) 1

[CLO92] David Cox, John Little, and Donal O'Shea, Ideals, varieties, and algorithms, Undergraduate Texts in Mathematics, Springer-Verlag, New York, 1992, An introduction to computational algebraic geometry and commutative algebra. MR MR1189133 (93j:13031) 4

[CoC] CoCoATeam, CoCoA: a system for doing Computations in Commutative Algebra, Available at http://cocoa.dima.unige.it. 1 
[CQR07] F. Chyzak, A. Quadrat, and D. Robertz, OreModules: A Symbolic Package for the Study of Multidimensional Linear Systems, Applications of TimeDelay Systems (J. Chiasson and J.-J. Loiseau, eds.), LNCIS 352, Springer, 2007, (http://wwwb.math.rwth-aachen.de/OreModules), pp. 233-264. 20

[CS98] Frédéric Chyzak and Bruno Salvy, Non-commutative elimination in Ore algebras proves multivariate identities, J. Symbolic Comput. 26 (1998), no. 2, 187-227. MR MR1635242 (99g:68103) 20,21

[DL06] Wolfram Decker and Christoph Lossen, Computing in algebraic geometry, Algorithms and Computation in Mathematics, vol. 16, Springer-Verlag, Berlin, 2006, A quick start using SINGULAR. MR MR2220403 (2007b:14129) 4

[Eis95] David Eisenbud, Commutative algebra, Graduate Texts in Mathematics, vol. 150, SpringerVerlag, New York, 1995, With a view toward algebraic geometry. MR MR1322960 (97a:13001) 8

[Fab07] Anna Fabianska, QuillenSuslin: A Maple package to compute a free basis of a projective module over the polynomial ring, (http://wwwb.math.rwth-aachen.de/QuillenSuslin), 20062007. 21

[FQ07] Anna Fabianska and Alban Quadrat, Applications of the Quillen-Suslin theorem in multidimensional systems theory, Proceedings of the Workshop on Gröbner bases in Control Theory and Signal Processing, Radon Book Series, de Gruyter, 2007, To appear. 21

[GB98a] Vladimir P. Gerdt and Yuri A. Blinkov, Involutive bases of polynomial ideals, Math. Comput. Simulation 45 (1998), no. 5-6, 519-541, Simplification of systems of algebraic and differential equations with applications. MR MR1627129 (99e:13033) 20

[GB98b] _ Minimal involutive bases, Math. Comput. Simulation 45 (1998), no. 5-6, 543-560, Simplification of systems of algebraic and differential equations with applications. MR MR1627130 (99e:13034) 20

[Ger05] Vladimir P. Gerdt, Involutive algorithms for computing Gröbner bases, Computational commutative and non-commutative algebraic geometry, NATO Sci. Ser. III Comput. Syst. Sci., vol. 196, IOS, Amsterdam, 2005, pp. 199-225. MR MR2179201 (2007c:13040) 20

[GM03] Sergei I. Gelfand and Yuri I. Manin, Methods of homological algebra, second ed., Springer Monographs in Mathematics, Springer-Verlag, Berlin, 2003. MR MR1950475 (2003m:18001) 17

[GP02] Gert-Martin Greuel and Gerhard Pfister, A singular introduction to commutative algebra, Springer-Verlag, Berlin, 2002, With contributions by Olaf Bachmann, Christoph Lossen and Hans Schönemann, With 1 CD-ROM (Windows, Macintosh, and UNIX). MR MR1930604 (2003k:13001) 5, 11, 12, 15

[GPS05] G.-M. Greuel, G. Pfister, and H. Schönemann, Singular 3.0, A Computer Algebra System for Polynomial Computations, Centre for Computer Algebra, University of Kaiserslautern, 2005, http://www.singular.uni-kl.de. 1

[GS] Daniel R. Grayson and Michael E. Stillman, Macaulay 2, a software system for research in algebraic geometry, Available at http://www.math.uiuc.edu/Macaulay2/. 1

[HS97] P. J. Hilton and U. Stammbach, A course in homological algebra, second ed., Graduate Texts in Mathematics, vol. 4, Springer-Verlag, New York, 1997. MR MR1438546 (97k:18001) 1, 18, 19

[KR00] Martin Kreuzer and Lorenzo Robbiano, Computational commutative algebra. 1, SpringerVerlag, Berlin, 2000. MR MR1790326 (2001j:13027) 4

[KR05] , Computational commutative algebra. 2, Springer-Verlag, Berlin, 2005. MR MR2159476 (2006h:13036) 8

[LS03] Viktor Levandovskyy and Hans Schönemann, PLURAL-a computer algebra system for noncommutative polynomial algebras, Proceedings of the 2003 International Symposium on 
Symbolic and Algebraic Computation (New York), ACM, 2003, pp. 176-183 (electronic). MR MR2035210 1

[MR01] J. C. McConnell and J. C. Robson, Noncommutative Noetherian rings, revised ed., Graduate Studies in Mathematics, vol. 30, American Mathematical Society, Providence, RI, 2001, With the cooperation of L. W. Small. MR MR1811901 (2001i:16039) 1

[PR05] W. Plesken and D. Robertz, Janet's approach to presentations and resolutions for polynomials and linear PDEs, Arch. Math. (Basel) 84 (2005), no. 1, 22-37. MR MR2106402 (2005h:13043) 20

[QR] A. Quadrat and D. Robertz, Computation of projective dimension and bases over the Weyl algebras, Accepted for publication. 21, 28

[Rob06] D. Robertz, Formal Computational Methods for Control Theory, Ph.D. thesis, RWTH Aachen, Germany, 2006, This thesis is available at http://darwin.bth.rwth-aachen.de/opus/volltexte/2006/1586. 20

[Rob07] Daniel Robertz, JanetOre: A Maple package to compute a JANET basis for modules over ORE algebras, 2003-2007, (http://wwwb.math.rwth-aachen.de:8040). 20

[Roo62] Jan-Erik Roos, Bidualité et structure des foncteurs dérivés de lim dans la catégorie des modules sur un anneau régulier, C. R. Acad. Sci. Paris 254 (1962), $\overrightarrow{1556-1558 . ~ M R ~ M R 0136639 ~(25 ~}$ \#106a) 2

[Rot79] Joseph J. Rotman, An introduction to homological algebra, Pure and Applied Mathematics, vol. 85, Academic Press Inc. [Harcourt Brace Jovanovich Publishers], New York, 1979. MR MR538169 (80k:18001) 1

[Sch80] Frank-Olaf Schreyer, Die Berechnung von Syzygien mit dem verallgemeinerten Weierstraßschen Divisionssatz und eine Anwendung auf analytische Cohen-Macaulay-Stellenalgebren minimaler Multiplizität, Diploma thesis, Univ. Hamburg, Germany, 1980. 4

[Sch03] Hal Schenck, Computational algebraic geometry, London Mathematical Society Student Texts, vol. 58, Cambridge University Press, Cambridge, 2003. MR MR2011360 (2004k:13001) 8

[Wei94] Charles A. Weibel, An introduction to homological algebra, Cambridge Studies in Advanced Mathematics, vol. 38, Cambridge University Press, Cambridge, 1994. MR MR1269324 (95f:18001) 1

[ZL02] E. Zerz and V. Lomadze, A constructive solution to interconnection and decomposition problems with multidimensional behaviors, SIAM J. Control Optim. 40 (2001/02), no. 4, 1072-1086 (electronic). MR MR1882725 (2002m:93020) 7

Lehrstuhl B für Mathematik, RWTH-Aachen University, 52062 Germany

E-mail address: mohamed.barakat@rwth-aachen.de, daniel@momo.math.rwth-aachen.de 